\title{
Integración del Pensamiento Computacional en la educación primaria y secundaria en Latinoamérica: una revisión sistemática de literatura
}

\author{
Integration of Computational Thinking in Elementary and \\ Secondary School in Latin America: A Systematic Literature \\ Review \\ Daniel Andrés Quiroz-Vallejo \\ Universidad de Antioquia. Medellín, Colombia. \\ daniel.quirozv@udea.edu.co \\ Jaime Andrés Carmona-Mesa \\ Universidad de Antioquia. Medellín, Colombia \\ jandres.carmona@udea.edu.co \\ Alexander Castrillón-Yepes \\ Universidad de Antioquia. Medellín, Colombia \\ alexander.castrillony@udea.edu.co \\ Jhony Alexander Villa-Ochoa \\ Universidad de Antioquia. Medellín, Colombia \\ jhony.villa@udea.edu.co
}

\section{Resumen}

El Pensamiento Computacional (PC) se ha consolidado como una línea de investigación internacional, sin embargo, en Latinoamérica son limitados los estudios que examinan su desarrollo e integración en la educación primaria y secundaria. El objetivo de este estudio fue analizar la integración del PC en la educación primaria y secundaria en Latinoamérica, a partir de una revisión sistemática de literatura de los estudios publicados entre 2006 y 2020. Los resultados se agruparon en dos categorías principales: las conceptualizaciones del PC y las estrategias usadas para la integración del PC. Se encontró que las conceptualizaciones se dividen en dos grupos: unas ligadas a las ciencias de la computación y otras en las que se entiende el PC como un recurso metodológico. Frente a las estrategias, se encontró que las más usadas en la región son la robótica educativa y la programación en lenguajes con bloques.

Palabras clave: pensamiento computacional, conceptualizaciones, estrategias, educación primaria y secundaria.

\section{Abstract}

Computational Thinking (CT) became established as a line of international research, however, in Latin America there are limited studies that examine its development and integration in elementary and secondary school. The objective of this study was to analyze the integration of CT in elementary and secondary 
school education in Latin America, based on a systematic literature review of studies published between 2006 and 2020. The results were grouped into two main categories: conceptualizations of CT and strategies to integrate CT. It was found that conceptualizations are divided into two groups, those related to computer science and others in which CT is understood as a methodological resource. Regarding strategies, it was found that educational robotics and programming in block languages are the most used in the region.

Keywords: computational thinking, conceptualizations, strategies, basic and secondary education.

\section{Introducción}

En la actualidad, las habilidades relacionadas con el Pensamiento Computacional (PC) se posicionan como fundamentales para desarrollarse en la sociedad del siglo XXI (Zhang y Nouri, 2019) y se constituyen como un tema de interés para la investigación y para el desarrollo de innovaciones e intervenciones en las clases de áreas STEM (Ciencias, Tecnología, Ingeniería y Matemáticas, por sus siglas en inglés) (Grover y Pea, 2018). EI PC se identifica como un recurso con potencial para solucionar problemas de maneras creativas (Carmona-Mesa et al. 2021) y una de sus definiciones más citadas refiere un proceso de pensamiento donde las soluciones se representan en una forma que puede ser llevada a cabo eficazmente por un agente procesador de información (Wing, 2011).

La integración de actividades para fomentar el PC en el sistema escolar tiene un amplio potencial para mejorar las habilidades cognitivas de los estudiantes (Huerta y Velásquez, 2021). De acuerdo con Cansu y Cansu (2019), estas actividades promueven en los estudiantes estrategias para comprender, interpretar y transformar su realidad. Si bien estas actividades tienden a desarrollarse mediante la creación de algoritmos computacionales o aspectos relacionados con las ciencias de la computación, el PC involucra un conjunto de habilidades útiles para todas las personas en la actualidad y no se limita solo a las ciencias de la computación (Wing, 2006).

Con los estudios de Papert (1980), se inició un movimiento que promovía el desarrollo de habilidades computacionales en los estudiantes de los diferentes niveles de escolaridad, sin embargo, fue a partir de los estudios de Wing (2006; 2008; 2011) que se adoptó la denominación de PC y se promovió su integración en los currículos escolares de Estados Unidos. Desde entonces, los estudios sobre el PC y su integración en el campo educativo han incrementado de manera considerable en diferentes partes del mundo. Por ejemplo, Tang et al. (2020) reportan que entre 2006 y 2012, el promedio de artículos publicados año a año en esta temática era de 36,1 , mientras que entre 2013 y 2018 , fue de 80,1 . Por su parte, Roig-Vila y Moreno-Isac (2020) ponen en evidencia que, en el campo de la educación, entre 2008 y 2018, también hubo un aumento significativo en la publicación de artículos, pasando de 1, en 2008, a 49, en 2018.

Aunque la definición que plantea Wing se ha popularizado en el campo, los debates frente a las definiciones operativas del PC en educación continúan abiertos (Bocconi et al., 2016; Zhang y Nouri, 2019; Roig-Vila y Moreno-Isac, 2020). De este modo, en la literatura internacional no existe una comprensión homogénea de este tipo de pensamiento, ni de

Integración del Pensamiento Computacional en la educación primaria y secundaria en Latinoamérica: una revisión sistemática de literatura. D. A. Quiroz-Vallejo, J.A. CarmonaMesa, A. Castrillón-Yepes y J.A. Villa-Ochoa. 
sus alcances y formas de integración en la cotidianidad escolar; entre ellas: estudio de algoritmos (Stephens y Kadijevich, 2019), habilidades para solucionar problemas (Denning, 2009) y pensar como los profesionales de la computación (Wing, 2006). Stephens y Kadijevich (2019), proponen que el área de estudio de los algoritmos puede enriquecerse a partir de conceptos de informática y matemáticas, siendo uno de ellos el PC, el cual definen como un pensamiento algorítmico apoyado por algún proceso de automatización; en este sentido, estos autores desarrollan una idea de PC altamente apoyada por las matemáticas. Denning (2009), sostiene que el PC puede entenderse como una serie de habilidades para la resolución de problemáticas del día a día, al mismo tiempo que cuestiona la comprensión de Wing (2006) en la que el PC refiere a pensar como un científico de la computación, dado que esta puede sugerir que el PC solo es relevante para el campo de las ciencias de la computación.

La diversidad de comprensiones, enfoques y propósitos acerca del PC sugiere diversos desafíos, no solo para la investigación, sino también para su integración en los diseños curriculares de educación primaria y secundaria (Grover y Pea, 2013), como en las universitarias (Villa-Ochoa y Castrillón-Yepes, 2020). Asimismo, aún existe una necesidad de articulación entre docencia e investigación y del desarrollo de innovaciones e intervenciones que se basen en resultados de investigación y de formación de profesores que participen de esos diseños y los gestionen en sus clases (Carmona-Mesa et al., 2022; Zampieri y Javaroni, 2020; Hsu et al., 2018; Lockwood y Mooney, 2018; Bocconi et al., 2016).

En Latinoamérica, se han llevado a cabo iniciativas para promover una integración del PC en la educación primaria y secundaria; por ejemplo, organizaciones como Microsoft tienen interés en ampliar las oportunidades para los jóvenes de la región en términos de empleo, emprendimiento y reingreso a la educación, además de apoyar a los ministerios de educación en la construcción de políticas educativas en las escuelas (Jara y Hepp, 2016). Aunque estos mismos autores resaltan que hay diversas organizaciones que apoyan procesos en los que se incorpora el PC a través de la programación, ponen de relieve la necesidad de que otras organizaciones se sumen a este tipo de proyectos, además de resaltar el desafío que enfrenta la región en cuanto al diseño de currículos pertinentes para integrar el PC y una adecuada formación a los docentes en esta temática.

En concordancia con lo anterior, Villa-Ochoa y Castrillón-Yepes (2020) ponen de manifiesto dos necesidades de investigación en la región; por un lado, la necesidad de que se desarrollen más estudios referentes al PC en áreas diferentes a la computación, y, por el otro lado, el desafío de desarrollar trabajos tanto teóricos como metodológicos que permitan integrar las distintas habilidades del PC en problemas interdisciplinares. Al respecto, Quiroz-Vallejo (2020) también sostiene que es necesario desarrollar proyectos que ofrezcan análisis profundos acerca del impacto del PC en los niveles de educación primaria y secundaria.

En sus revisiones Curasma y Curasma (2020) y Huerta y Velásquez (2021), aportan a la investigación en educación primaria y secundaria respecto al PC en Latinoamérica. Los primeros a través de un estudio enfocado en las políticas educativas, las metodologías de enseñanza, los lenguajes y recursos usados y las técnicas de evaluación; y los segundos, a través del análisis del PC como una habilidad genérica para la resolución de problemas

Integración del Pensamiento Computacional en la educación primaria y secundaria en Latinoamérica: una revisión sistemática de literatura. D. A. Quiroz-Vallejo, J.A. CarmonaMesa, A. Castrillón-Yepes y J.A. Villa-Ochoa.

Página 3 de 33 
reales en distintos campos del conocimiento. Sin embargo, aún existe la necesidad de identificar las conceptualizaciones del PC que circulan en la región y, en correspondencia con ello, las maneras de integrarlo en las diferentes disciplinas dentro de las instituciones educativas. En este sentido, el objetivo de este artículo es analizar la integración del PC en la educación primaria y secundaria en Latinoamérica a través de una revisión sistemática de literatura.

El resto de este artículo está organizado de la siguiente manera: en la sección metodología se describen las preguntas de investigación y el proceso llevado a cabo para seleccionar los artículos de la revisión. En la sección de resultados se presenta el análisis de la información descriptiva de los artículos revisados; la integración del PC como recurso metodológico y como componente de las ciencias de la computación, además de las estrategias usadas para su inclusión. Finalmente, en las secciones discusión y conclusiones se interpretan los resultados obtenidos a partir del proceso de revisión y se declaran oportunidades de trabajo a futuro para la región en cuanto a la integración del PC en educación primaria y secundaria.

\section{Metodología}

Con el objetivo de analizar la integración del PC en la educación primaria y secundaria en Latinoamérica, se realizó una revisión sistemática de literatura (Wohlin et al., 2012). Para esto se delimitaron dos dimensiones: sus conceptualizaciones y las estrategias usadas para la implementación; además, se siguieron las fases detalladas por Wohlin et al. (2012) para una revisión. Las preguntas que guiaron la revisión son:

- ¿Qué conceptualizaciones frente al Pensamiento Computacional se presentan en estudios de educación primaria y secundaria en Latinoamérica?

- ¿Qué tipo de estrategias son utilizadas en la educación primaria y secundaria para promover el Pensamiento Computacional en Latinoamérica?

Con estas preguntas, el protocolo de revisión se desarrolló a partir de una ecuación de búsqueda en inglés y en español (Tabla 1.) que se puso en marcha en las bases de datos Scopus, Eric, Scielo, Dialnet, Redalyc, IEEE Xplore y ACM, las cuales fueron seleccionadas por ser fuentes de datos confiables, con impacto tanto en Latinoamérica como a nivel internacional y algunas ser especializadas en la temática de interés.

Tabla 1.

Ecuaciones de búsqueda

\begin{tabular}{|c|c|}
\hline Español & Inglés \\
\hline $\begin{array}{l}\text { ("pensamiento computacional”) AND } \\
\text { ("educación básica" OR "primaria" OR } \\
\text { "secundaria" OR "bachillerato" OR } \\
\text { "educación media" OR “K-*”) }\end{array}$ & $\begin{array}{l}\text { ("computational thinking") AND ("basic education" } \\
\text { OR "elementary school" OR "K-"” OR "high school" } \\
\text { OR "middle school" OR "secondary education" OR } \\
\text { "primary education" OR "compulsory education") }\end{array}$ \\
\hline
\end{tabular}

Elaboración de los autores

Integración del Pensamiento Computacional en la educación primaria y secundaria en Latinoamérica: una revisión sistemática de literatura. D. A. Quiroz-Vallejo, J.A. CarmonaMesa, A. Castrillón-Yepes y J.A. Villa-Ochoa. 
De manera particular, en las bases de datos Scielo y Redalyc hubo variaciones en los resultados al iterar el término $\mathrm{K}-{ }^{*}$, por K-9 y K-12, dando un incremento en los resultados; en Scielo se obtuvieron dos resultados más en la búsqueda en español y en Redalyc, 5 resultados más en la búsqueda en el mismo idioma. Además de lo anterior, los criterios de inclusión y exclusión de documentos consideraron que estos tuviesen evaluación de pares y que se evidenciara una conceptualización, metodología o estrategia para el desarrollo del PC en Latinoamérica, desde el año 2006 hasta el 2020, inclusive. Estas fechas fueron elegidas teniendo en cuenta que, a partir del año 2006 los estudios frente al pensamiento computacional comenzaron a ampliarse en diferentes partes del mundo. En la Tabla 2 se describen en totalidad los criterios de inclusión y exclusión para la búsqueda de documentos. Por otra parte, en la Tabla 3 se muestran los resultados de la revisión por base de datos e idioma.

\section{Tabla 2.}

\section{Criterios de inclusión y exclusión}

\begin{tabular}{ll}
\hline Criterios de inclusión & Criterios de exclusión \\
\hline $\begin{array}{l}\text { Artículos o capítulos de libros con evaluación de } \\
\text { pares. }\end{array}$ & $\begin{array}{l}\text { Memorias de eventos académicos, } \\
\text { tesis y literatura gris. }\end{array}$ \\
$\begin{array}{l}\text { Documentos enfocados en los niveles de educación } \\
\text { primaria o secundaria (6-18 años). }\end{array}$ & $\begin{array}{l}\text { Documentos enfocados en niveles de } \\
\text { educación diferentes a educación } \\
\text { primaria, secundaria o K-12. }\end{array}$ \\
$\begin{array}{l}\text { Estudios empíricos en educación primaria y } \\
\text { secundaria o K-12. }\end{array}$ & $\begin{array}{l}\text { Ensayos, estudios teóricos o revisiones } \\
\text { de literatura. }\end{array}$ \\
$\begin{array}{l}\text { Estudios cuyo trabajo empírico se desarrolla en } \\
\text { Latinoamérica }\end{array}$ & $\begin{array}{l}\text { Estudios que fueron desarrollados en } \\
\text { países que no son de Latinoamérica. } \\
\text { Documentos en los que no se explícita }\end{array}$ \\
$\begin{array}{l}\text { Estudios que describen conceptualizaciones, } \\
\text { metodologías o estrategias para integrar el } \\
\text { pensamiento computacional en la educación primaria } \\
\text { y secundaria. }\end{array}$ & $\begin{array}{l}\text { la metodología, cursos o } \\
\text { comprensiones del PC. }\end{array}$ \\
$\begin{array}{l}\text { Documentos que están escritos en } \\
\text { idiomas diferentes al inglés, español o }\end{array}$ \\
portugués.
\end{tabular}

Elaboración de los autores

La búsqueda en las bases de datos se realizó el 29 de marzo de 2021, esta arrojó como resultado 5320 documentos. Se eliminaron los duplicados (doce documentos) y se hizo una lectura de títulos, resúmenes y palabras clave, y se aplicaron los criterios de inclusión y exclusión, de lo cual arrojó como resultado 76 documentos. Luego, se hizo una primera lectura del contenido de los documentos y se aplicaron nuevamente los criterios de inclusión y exclusión, lo cual arrojó como resultado 28 documentos para el análisis a profundidad en la revisión.

Integración del Pensamiento Computacional en la educación primaria y secundaria en Latinoamérica: una revisión sistemática de literatura. D. A. Quiroz-Vallejo, J.A. CarmonaMesa, A. Castrillón-Yepes y J.A. Villa-Ochoa. 
Cada uno de los 28 documentos se revisó de manera detallada con el fin de identificar y codificar información descriptiva, las conceptualizaciones explícitas o implícitas del PC en ellos y las estrategias usadas para su integración en la educación primaria y secundaria. En particular, el criterio de exclusión que más destacó en la selección de los estudios a analizar fue el de ubicación geográfica. Si bien este criterio no fue incluido como palabra clave en las ecuaciones de búsqueda, se revisó con detalle la procedencia de cada investigación revisada y se encontró que la mayoría de los documentos no eran de esta región. En la Tabla 3 se describe el proceso de selección por base de datos e idioma.

\section{Tabla 3.}

Resultados por bases de datos e idioma

\begin{tabular}{ccccc}
\hline & \multicolumn{2}{c}{ Búsqueda en inglés } & \multicolumn{2}{c}{ Búsqueda en español } \\
Base de datos & $\begin{array}{c}\text { Cantidad de } \\
\text { documentos } \\
\text { encontrados }\end{array}$ & $\begin{array}{c}\text { Seleccionados } \\
\text { primera ronda }\end{array}$ & $\begin{array}{c}\text { Cantidad de } \\
\text { documentos } \\
\text { encontrados }\end{array}$ & $\begin{array}{c}\text { Seleccionados } \\
\text { primera ronda }\end{array}$ \\
\hline Scopus & 99 & 5 & 1 & 0 \\
Eric & 1447 & 5 & 584 & 3 \\
Dialnet & 46 & 7 & 67 & 13 \\
Scielo & 1 & 1 & 3 & 1 \\
IEEE Xplore & 197 & 23 & 0 & 6 \\
Redalyc & 3530 & 7 & 29 & 0 \\
ACM & 1935 & 12 & 1 & 6 \\
\hline
\end{tabular}

Elaboración de los autores

Además de la información descriptiva, para el análisis se organizaron dos categorías correspondientes con las preguntas de investigación, a saber: las conceptualizaciones del PC y las estrategias para su fomento en la educación primaria y secundaria.

Las conceptualizaciones del PC son un tema de estudio con importancia para su integración en los currículos de los distintos niveles educativos. De acuerdo con Fessakis et al. (2018), las experiencias de implementación proveen retroalimentación que permite generar una revisión crítica de la integración del PC en educación primaria y secundaria. Por otra parte, si bien esto ha sido ampliamente analizado a nivel internacional (Shute et al., 2017; Bocconi et al., 2016), en Latinoamérica aún quedan elementos por explorar en este sentido. De este modo, la categoría de conceptualizaciones del PC refiere a las interpretaciones implícitas y explícitas que se reflejan de la temática en la literatura revisada. En particular, se analizan las conceptualizaciones del PC al ser usado como recurso metodológico para desarrollar habilidades en las disciplinas y asignaturas de las 
instituciones educativas y al ser usado como un componente de las ciencias de la computación. Para ello se codificaron las definiciones, habilidades y conceptos del PC y sus relaciones entre las ciencias de la computación y las otras áreas ( $p$. ej. pensamiento matemático y analítico).

Las estrategias para integrar el PC se comprenden como los distintos recursos de enseñanza, lenguajes de programación, robótica, juegos de mesa, entre otras, usados en las distintas experiencias de integración del PC que aportan a que los estudiantes aprendan a dominar las diferentes habilidades relacionadas con el PC (Xu, 2018). El conocimiento de estas estrategias es útil puesto que ofrecen información importante acerca de las tendencias de instrucción a usar para enseñar el PC y sus comprensiones por parte de profesores y estudiantes (Kale et al., 2018). Además, estas estrategias se consolidan como información relevante para el diseño de ambientes que promuevan el aprendizaje o el desarrollo de competencias y habilidades en los contextos escolares.

Para evaluar la fiabilidad entre evaluadores respecto a la selección de los estudios, dos de los autores codificaron, de manera independiente, una muestra de 10 de los 76 artículos iniciales con base en los criterios de inclusión y exclusión descritos en la Tabla 2. La fiabilidad entre los autores fue medida a través del coeficiente kappa de Cohen y su resultado fue $k=0.74$, lo que, de acuerdo con McHugh (2012), representa un acuerdo substancial entre autores. En la Tabla 4 se muestra el proceso detallado que fue seguido para calcular la fiabilidad entre evaluadores; en ella, $p_{0}$ es el acuerdo relativo observado entre los autores, sí indica que el evaluador incluye el documento bajo los criterios establecidos y no indica que el evaluador no incluye el documento, $p_{e}$ es la probabilidad hipotética de acuerdo casual y $k$ el coeficiente kappa de Cohen.

Tabla 4.

Fiabilidad entre evaluadores

\begin{tabular}{|c|c|c|c|c|c|}
\hline & \multicolumn{2}{|c|}{ Autor 1} & \multicolumn{2}{|c|}{ Autor 2} & Resultado \\
\hline \multirow[b]{2}{*}{$p_{0}$} & Sí & No & Sí & No & 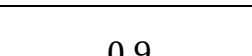 \\
\hline & $8 / 10$ & $2 / 10$ & $7 / 10$ & $3 / 10$ & 0.9 \\
\hline $\begin{array}{l}\text { Probabilidad de que los } \\
\text { dos autores evalúen sí } \\
\text { casualmente }\end{array}$ & \multicolumn{2}{|c|}{$\frac{8}{10}=0.8$} & \multicolumn{2}{|c|}{$\frac{7}{10}=0.7$} & $0.8 * 0.7=0.56$ \\
\hline $\begin{array}{l}\text { Probabilidad de que los } \\
\text { autores evalúen no } \\
\text { casualmente }\end{array}$ & \multicolumn{2}{|c|}{$\frac{2}{10}=0.2$} & \multicolumn{2}{|c|}{$\frac{3}{10}=0.3$} & $0.3 * 0.2=0.06$ \\
\hline$p_{e}$ & \multicolumn{4}{|c|}{$0.56+0.06$} & 0.62 \\
\hline$k$ & \multicolumn{4}{|c|}{$k=\frac{p_{0}-p_{e}}{1-p_{e}}=\frac{0.9-0.62}{1-0.62}$} & 0.74 \\
\hline
\end{tabular}

Elaboración de los autores

Integración del Pensamiento Computacional en la educación primaria y secundaria en Latinoamérica: una revisión sistemática de literatura. D. A. Quiroz-Vallejo, J.A. CarmonaMesa, A. Castrillón-Yepes y J.A. Villa-Ochoa. 


\section{Resultados}

Los resultados de esta revisión se presentan en dos secciones: primero, la información descriptiva de los estudios revisados y, segundo, las categorías de análisis declaradas en la metodología (conceptualizaciones y estrategias).

\section{Distribución de artículos en países, años de publicación, áreas y tiempo de implementación}

En esta sección se presenta la información relacionada con los países de Latinoamérica en los que se identificaron las investigaciones revisadas, la duración de las experiencias relacionadas con el PC, las áreas del conocimiento en las que se prioriza su integración en los sistemas escolares y los años de publicación de estas experiencias.

\section{Países}

Las experiencias reportadas en los documentos revisados se distribuyeron en nueve países de Latinoamérica, Brasil como líder con trece publicaciones, seguido por México y Colombia con tres cada uno; luego, en República Dominicana, Uruguay y Argentina se encontraron dos reportes de experiencias acerca de integración del PC en la educación primaria y secundaria en cada uno de estos países; mientras que en Chile, Perú y Ecuador se registra una publicación por cada país. En la Figura 1 se presenta esta información de forma gráfica.

\section{Duración de las experiencias}

Las experiencias para la integración del PC reportadas en esta revisión son investigadas a lo largo de períodos concretos. En este sentido, se tiene en cuenta, en este apartado, la cantidad de tiempo que duró la implementación de las actividades relacionadas con el PC en las distintas investigaciones y se relacionan con las metodologías empleadas en los distintos estudios. En la Tabla 5 se relaciona la duración de las experiencias con el tipo de estudio realizado.

Los resultados frente a la duración de las experiencias y su relación con los tipos de estudio permiten identificar que las experiencias para la integración del PC han sido estudiadas de manera mayoritaria a través de investigaciones cualitativas, en las cuales se presentan los estudios de caso como la alternativa más usada. Por su parte, las investigaciones cuantitativas se enfocaron en diseños experimentales y cuasiexperimentales, mientras que solo uno de los documentos reportó el uso de métodos mixtos a través de un estudio de caso. 
RED. Revista de Educación a Distancia. Núm. 68, Vol. 21. Artíc. 7, 30-Nov-2021

DOI: http://dx.doi.org/10.6018/red.485321

\section{Figura 1.}

Distribución de documentos por países

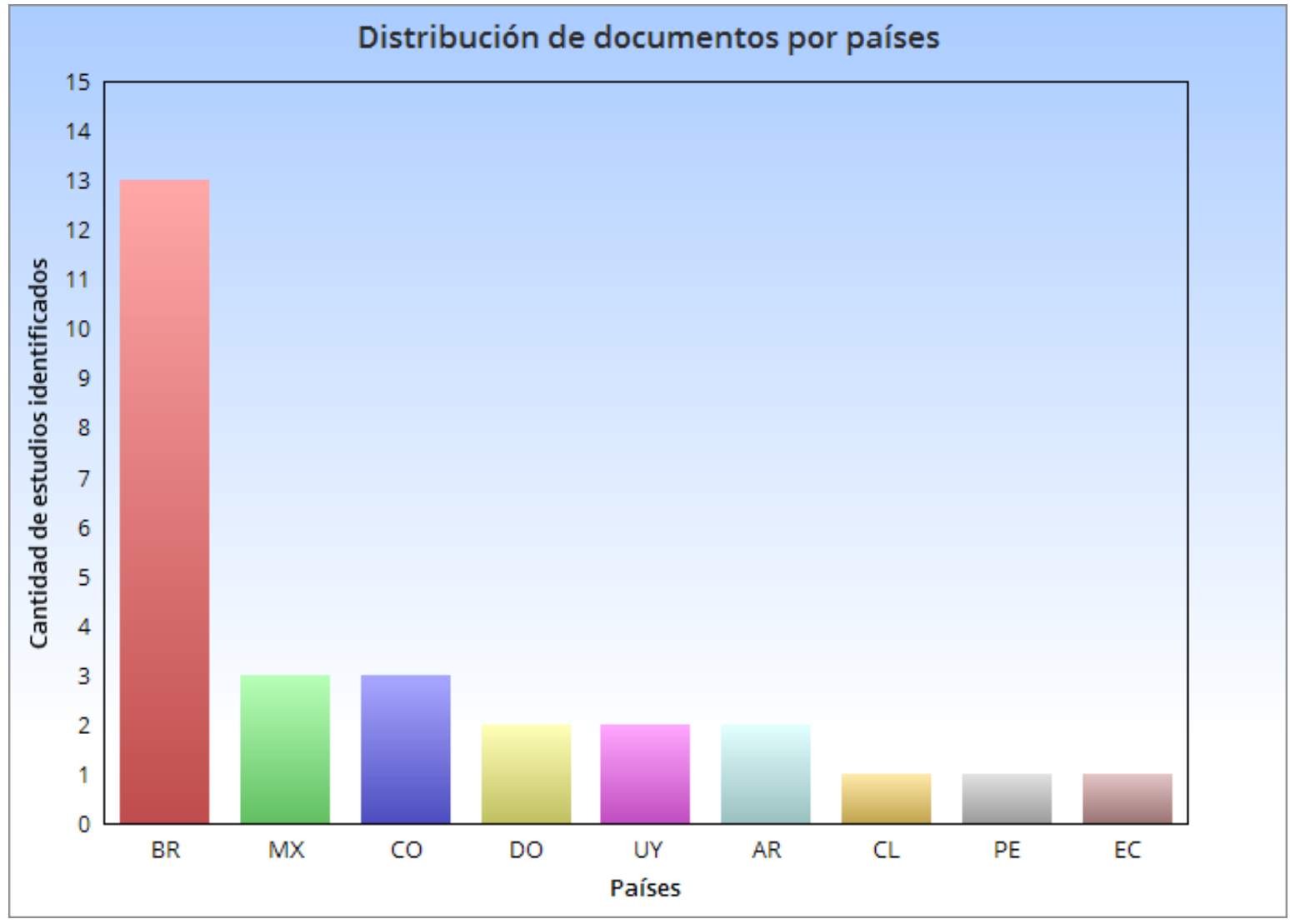

Elaboración de los autores

Tabla 5.

Tipos de estudio y duración de experiencias

\begin{tabular}{lcccc}
\hline & $\begin{array}{c}\text { Menos de dos } \\
\text { meses }\end{array}$ & $\begin{array}{c}\text { Entre tres y seis } \\
\text { meses }\end{array}$ & $\begin{array}{c}\text { Entre seis meses } \\
\text { y un año }\end{array}$ & Mayor a un año \\
\hline Cualitativo & 7 & 4 & 3 & 5 \\
Cuantitativo & 4 & 2 & 0 & 2 \\
Mixto & 1 & 0 & 0 & 0 \\
\hline
\end{tabular}

Elaboración de los autores

La cantidad de experiencias para la integración del PC en las aulas de clase con una duración menor a seis meses representa el 64\% (dieciocho de veintiocho) del total de los estudios revisados. Además, la generalidad de los estudios se centra en investigar a profundidad casos específicos con grupos de estudiantes reducidos. En este sentido, la integración a largo plazo y de manera continuada en los diferentes currículos escolares, se ve aún limitada en la región por la carencia de investigaciones de mayor duración y con un alcance de población amplio que permitan extraer elementos generales para apoyar la integración del PC en los planes educativos.

Integración del Pensamiento Computacional en la educación primaria y secundaria en Latinoamérica: una revisión sistemática de literatura. D. A. Quiroz-Vallejo, J.A. CarmonaMesa, A. Castrillón-Yepes y J.A. Villa-Ochoa. 
RED. Revista de Educación a Distancia. Núm. 68, Vol. 21. Artíc. 7, 30-Nov-2021

DOI: http://dx.doi.org/10.6018/red.485321

\section{Áreas del sistema escolar priorizadas}

En cuanto a las disciplinas en las cuales se desarrollan experiencias para integrar el PC, los resultados muestran que las clases de informática/tecnología (diecinueve estudios) y matemáticas (siete estudios) tienen la mayor frecuencia de implementación de estrategias relativas al PC. En el área de informática/tecnología, principalmente, se observaron actividades relacionadas con la creación de algoritmos computacionales en lenguajes de programación en los cuáles la escritura del código es necesaria, como Python o $\mathrm{C}_{++}$, y solo uno de los estudios integró una estrategia desenchufada a través de un juego de mesa; en matemáticas predominaron actividades de resolución de problemas donde las habilidades del PC eran un medio para el estudio de contenidos matemáticos y, en menor medida, hubo diseños para el estudio de algoritmos y el uso de las matemáticas para resolver problemas o comprender contextos de la computación.

Por otra parte, se registraron experiencias (dos estudios) que integran el PC en las clases de humanidades, en las cuales el diseño de videojuegos y el storytelling fueron el foco de enseñanza para el desarrollo de conocimientos tanto de historia como de PC. Con estos hallazgos se evidencia que, en la actualidad, la integración del PC se presenta principalmente en las áreas relacionadas con las ciencias de la computación, a pesar de reconocerse su importancia en otros campos disciplinares (Villa-Ochoa y Castrillón-Yepes, 2020). En este sentido, la integración del PC en asignaturas diferentes a las relacionadas con las ciencias de la computación sigue constituyéndose como un reto en la región.

\section{Año de publicación.}

Con relación al año de publicación de los estudios, aunque la revisión contempló 15 años en sus criterios de inclusión, entre 2006 y 2020, no se registraron estudios en la búsqueda en los primeros 8 años, los resultados solo se encontraron a partir del año 2013, año desde el cual la tendencia de publicación de experiencias para la integración del PC en la educación primaria y secundaria comenzó a aumentar en la región de manera sostenida. En la Figura 2 se muestra la tendencia de crecimiento de las publicaciones revisadas desde 2013 hasta 2020.

En esta sección, se describió la distribución de los artículos revisados en función de los países donde se registraron las experiencias, el tiempo de implementación, los tipos de estudio, las áreas priorizadas para la implementación de las experiencias de PC y los años de publicación. En este sentido, se identifica que la mayoría de los estudios son cualitativos y con una duración menor a seis meses en su implementación. Además, se priorizan las áreas de tecnología/informática para la integración del PC en la educación primaria y secundaria. Estos hallazgos permiten identificar potenciales rutas de acción para impulsar la investigación de la temática en la región; por ejemplo, la pertinencia de desarrollar más trabajos que tengan en cuenta experiencias de larga duración y con mayor población para dar cuenta de cuáles son las habilidades del PC que se desarrollan en el largo plazo para los estudiantes. Por otra parte, el uso del PC como recurso metodológico para apoyar los procesos interdisciplinares 0 de enseñanza en disciplinas diferentes a la informática/tecnología emerge como una posibilidad poco explorada que puede ampliarse

Integración del Pensamiento Computacional en la educación primaria y secundaria en Latinoamérica: una revisión sistemática de literatura. D. A. Quiroz-Vallejo, J.A. CarmonaMesa, A. Castrillón-Yepes y J.A. Villa-Ochoa. 
RED. Revista de Educación a Distancia. Núm. 68, Vol. 21. Artíc. 7, 30-Nov-2021 DOI: http://dx.doi.org/10.6018/red.485321

en la región. En la siguiente sección se presentan los resultados de dos categorías más en el análisis de la información: conceptualizaciones del PC y estrategias usadas para su integración.

\section{Figura 2.}

Distribución de documentos por año de publicación, entre 2013 y 2020

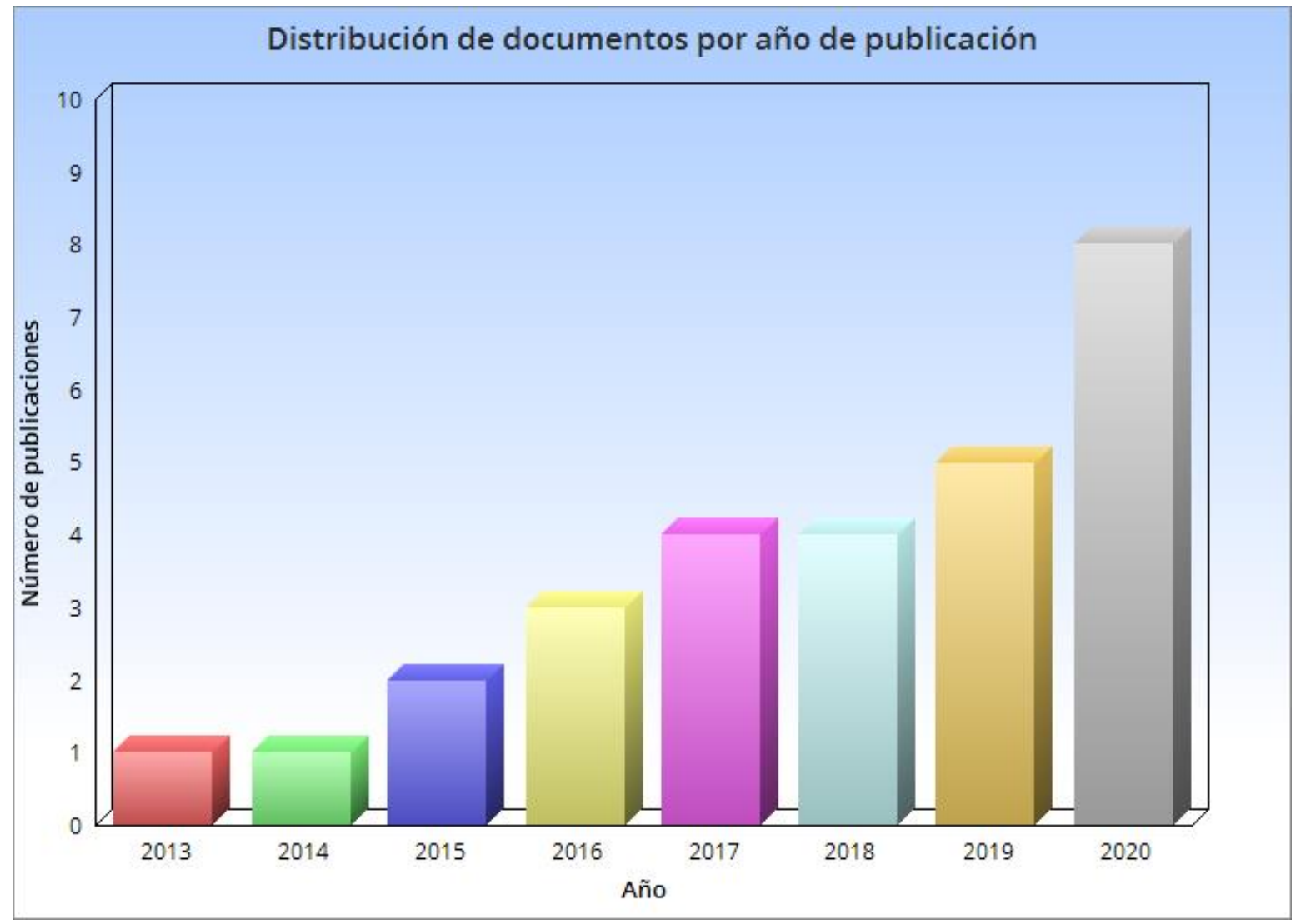

Elaboración de los autores

\section{Conceptualizaciones y estrategias}

Con relación a las categorías de análisis (conceptualizaciones y estrategias), en la Tabla 6 se presenta un resumen de los resultados que reporta la revisión en cada una, junto con algunos de los estudios relevantes en cada subcategoría. Además, la tabla presenta una relación entre las conceptualizaciones del PC y las estrategias usadas para implementarlo. Frente a las conceptualizaciones se identificaron dos subcategorías importantes: las conceptualizaciones para el uso del PC como recurso metodológico, es decir, como un medio para la enseñanza de las distintas disciplinas, y las conceptualizaciones del PC como un conjunto de habilidades propias de las ciencias de la computación, usadas de manera específica en las clases de informática/tecnología. En este sentido, en la tabla también se muestran las estrategias para la integración del PC que se reportan en la revisión de literatura y en qué tipo de conceptualización del PC tienden a enmarcarse. 
RED. Revista de Educación a Distancia. Núm. 68, Vol. 21. Artíc. 7, 30-Nov-2021

DOI: http://dx.doi.org/10.6018/red.485321

Tabla 6.

Relación entre conceptualizaciones del PC y estrategias usadas para su integración

\section{Conceptualizaciones del PC}

- Enfoque o conjunto de habilidades de resolución de problemas complejos (de Jesus y Silveira, 2020; ManriqueLosada et al., 2020; von Wangenheim et

Recurso metodológico en distintas disciplinas

Ciencias de la computación al., 2017; Costa et al., 2017)

- Habilidades de trabajo colaborativo (Basogain y Olmedo, 2020; de Souza et al., 2014)

- PC como recurso para fomentar la interdisciplinariedad (Corzo et al., 2020; Curasma et al., 2019; von Wangenheim et al., 2017; Costa et al., 2017)

- Creación de programas o algoritmos (da Silva et al., 2020; Kaminski y Boscarioli, 2018)

- Aplicación de nuevos métodos computacionales en problemas (de Jesus y Silveira, 2020; Manrique-Losada et al., 2020; Curasma et al., 2019; von Wangenheim et al., 2019)

- Reformulación de problemas para ser solucionados con estrategias computacionales (Schneider et al., 2020; da Silva et al., 2020; Barbosa y Maltempi, 2019; Araujo et al., 2018; González et al., 2018; Basogain et al., 2016; Enriquez et al., 2016)

\section{Estrategias para integrar el PC}

- Integración del PC a través de la Robótica Educativa (Curasma et al., 2019; Souza et al., 2019; Rosales et al., 2017; García, 2015)

- Integración del PC a través de la creación de juegos digitales (da Silva et al., 2020; Barbosa y Maltempi, 2019; Kaminski y Boscarioli, 2018; von Wangenheim et al., 2017)

- Integración del PC a través de la programación de algoritmos computacionales (da Silva et al., 2020; González et al., 2018; Araujo et al., 2018)

- Integración del PC a través de actividades desenchufadas (von Wangenheim et al., 2019)

\section{Elaboración de los autores}

\section{Conceptualizaciones del Pensamiento Computacional.}

La categoría conceptualizaciones del PC refiere a las interpretaciones implícitas y explícitas que se reflejan en las investigaciones revisadas de la temática. En este sentido, los resultados que se agrupan en esta categoría brindan información respecto a la influencia que tienen las diferentes perspectivas del PC en el diseño y desarrollo de experiencias educativas que predominan en la región. Esta categoría fue dividida en dos subcategorías para el análisis: las conceptualizaciones del PC como recurso metodológico y las conceptualizaciones del PC para las ciencias de la computación.

Integración del Pensamiento Computacional en la educación primaria y secundaria en Latinoamérica: una revisión sistemática de literatura. D. A. Quiroz-Vallejo, J.A. CarmonaMesa, A. Castrillón-Yepes y J.A. Villa-Ochoa. 
RED. Revista de Educación a Distancia. Núm. 68, Vol. 21. Artíc. 7, 30-Nov-2021

DOI: http://dx.doi.org/10.6018/red.485321

\section{PC como recurso metodológico.}

El desarrollo del PC como recurso metodológico, se refiere a una comprensión de este pensamiento como un vehículo o medio para alcanzar fines en las disciplinas o de relaciones entre ellas. Esta temática es clave puesto que permite reconocer los alcances y usos del PC en las asignaturas de las ciencias de la computación y otras asignaturas escolares. En este sentido, esta categoría describe las experiencias frente a la integración del PC con la finalidad de aportar al desarrollo de habilidades como la resolución de problemas, el trabajo colaborativo, así como el potencial del PC como un recurso metodológico que promueve la interdisciplinariedad.

Los resultados de la revisión muestran que, en el uso del PC como recurso metodológico, el más común es la del PC como un enfoque o conjunto de habilidades para la resolución de problemas complejos. En las experiencias en las que se conceptualiza el PC de esta manera, se promueven habilidades como el pensamiento crítico, la innovación, la abstracción y el uso de procesos sistémicos para optimizar las soluciones a los problemas propuestos. Por ejemplo, de Jesus y Silveira (2020) usan el PC como un recurso metodológico para automatizar las soluciones a problemáticas que proponían a los estudiantes a través de la creación de juegos digitales. Por otra parte, es común a los estudios que adoptan esta comprensión del PC, que la implementación de las experiencias sea desde los primeros años de escolaridad. Es el caso de la experiencia desarrollada por von Wangenheim et al. (2017), la cual se enfocó en la integración del PC de forma transversal en un curso de historia para niños entre 8 y 14 años. Así mismo, Costa et al. (2017) reportan un estudio en las clases de matemáticas a través de un enfoque de resolución de problemas matemáticos que se adecuaron para fortalecer las habilidades relacionadas con el $\mathrm{PC}$.

Por otra parte, existen conceptualizaciones del PC que ubican esta temática como un recurso para el desarrollo de habilidades de trabajo colaborativo, como la tolerancia y respeto entre compañeros de equipo. Por ejemplo, de Souza et al. (2014) reportaron experiencias en las que, a través del uso de la programación en equipos, los estudiantes manifestaron comunicarse mejor entre sí, dado que adquirieron mayores habilidades de comunicación asertiva. Asimismo, trabajos como los de Basogain y Olmedo (2020) muestran la posibilidad de usar el PC como un elemento para promover competencias relacionadas con la construcción de ciudadanía, a través del aprendizaje colaborativo online en escuelas de educación primaria en Uruguay.

Conforme se enunció antes, las matemáticas se constituyen como la segunda área en la cual se encontraron más estudios relacionados con el PC. Por esta razón, se reportan estudios que conceptualizan el PC para su uso en la educación matemática a través de procesos sistémicos y de combinación de algoritmos computacionales y matemáticos. Al respecto, Barbosa y Maltempi (2019) describen una experiencia que se enfocó en el apoyo a estudiantes que tenían dificultades con la comprensión de las ecuaciones de primer grado, a través del diseño de un juego digital en Scratch. La robótica educativa y el PC también fueron usados para complementar las clases de matemáticas en educación secundaria con resultados positivos, dado que las calificaciones de final de año de los

Integración del Pensamiento Computacional en la educación primaria y secundaria en Latinoamérica: una revisión sistemática de literatura. D. A. Quiroz-Vallejo, J.A. CarmonaMesa, A. Castrillón-Yepes y J.A. Villa-Ochoa.

Página 13 de 33 
estudiantes mejoraron y, en general, su comprensión de conceptos matemáticos tuvo un mayor alcance con relación a antes de las experiencias (Souza et al., 2019; Rodrigues et al., 2016). Por otra parte, Basogain et al. (2016), aunque no integran el PC directamente en cursos de matemáticas, reportan que esta temática y su comprensión a partir de procesos sistémicos, aporta de manera directa al desarrollo de competencias matemáticas en la educación primaria, como la comprensión y comunicación de problemas de forma organizada y basándose en la lógica.

Se reportan en la revisión, experiencias en las cuales las conceptualizaciones del PC tienden a ubicar la temática como un puente entre distintas disciplinas. En este sentido, Curasma et al. (2019) proponen que las habilidades que se derivan de la integración del PC son fundamentales para diferentes áreas al mismo tiempo, como las ciencias de la computación, las matemáticas, las ciencias sociales y las humanidades. Por esta razón, el PC se comprende de manera implícita como un recurso metodológico que promueve procesos interdisciplinares. Al respecto, se destacan experiencias en las que se integraron de forma transversal disciplinas como la historia y las ciencias de la computación al fomentar el PC por medio de la creación de juegos digitales con contenidos históricos (von Wangenheim et al. 2017). Por otro lado, Costa et al. (2017) proponen que los elementos del PC pueden fomentarse a través de distintas disciplinas, por lo que no es necesario hacer espacio en los currículos para nuevas asignaturas enfocadas solamente en el PC. Estos mismos autores reportan que lo anterior tiene el potencial de funcionar, especialmente, en los procesos interdisciplinares entre matemáticas y ciencias de la computación.

Con relación a lo anterior, estudios como los de Azevedo y Maltempi (2020) muestran cómo elementos de las ciencias de la computación pueden aportar a la enseñanza de las matemáticas a través del diseño de juegos digitales y dispositivos robóticos con Scratch, por parte de estudiantes de educación media. Estos autores dan cuenta de que esta experiencia de integración del PC de modo interdisciplinar favorece que los estudiantes adquieran habilidades como la resolución de problemáticas reales, la sistematización de conceptos para ser usados en situaciones concretas y el trabajo creativo; en este sentido, de manera implícita se mantiene la conceptualización del PC como un enfoque de resolución de problemáticas complejas. En la misma línea de ideas, Corzo et al. (2020) también integraron el PC de forma interdisciplinar entre las matemáticas y las ciencias de la computación, a través del enfoque STEAM en la educación básica. Sus resultados dan cuenta de que esta estrategia hace posible lograr los objetivos de aprendizaje propuestos en los currículos de matemáticas y amplía la capacidad de los estudiantes para relacionarse con las tecnologías digitales.

En síntesis, el PC como recurso metodológico se presentó en distintos escenarios en los que tuvo un rol de vehículo para obtener un fin. Esta conceptualización aportó en los estudios para desarrollar contenidos en disciplinas distintas a la informática/tecnología, razón por la cual se observa un potencial alto como recurso para desarrollar procesos interdisciplinares en los cuales el PC es el medio que ofrece el fortalecimiento de habilidades para la resolución de problemas y trabajo colaborativo entre pares. Además, esta conceptualización destaca por su diversidad de alternativas para aproximarse a distintas situaciones del sistema escolar, como las necesidades de refuerzos en algunas

Integración del Pensamiento Computacional en la educación primaria y secundaria en Latinoamérica: una revisión sistemática de literatura. D. A. Quiroz-Vallejo, J.A. CarmonaMesa, A. Castrillón-Yepes y J.A. Villa-Ochoa. 
clases o el diseño de proyectos conjuntos entre distintas asignaturas, lo que le permite trascender tanto las ciencias de la computación como las diferentes disciplinas y apoyar, de cara a algunos de los retos que tienen, los sistemas educativos de la región como la integración de tecnologías digitales y el desarrollo de las habilidades que son demandadas actualmente por la sociedad.

\section{PC como componente de las ciencias de la computación}

Conforme fue evidenciado en el apartado anterior, el PC puede ser usado para complementar la formación en distintas disciplinas enmarcadas en los currículos escolares. Sin embargo, sus comprensiones suelen retomarse, principalmente, de las ciencias de la computación. En este sentido, esta categoría retoma los estudios en los cuales el PC es una meta o un fin para alcanzar, a través del desarrollo de habilidades relacionadas directamente con las ciencias de la computación. Asimismo, en las conceptualizaciones del PC como componente de las ciencias de la computación, se reconoce la temática como una alternativa para el desarrollo de nuevos métodos computacionales, para la solución de problemas y la reformulación de problemas para la aplicación de estrategias computacionales.

Las experiencias revisadas que se enmarcan en el PC como un componente de las ciencias de la computación, hacen uso de la creación de programas de computadora para favorecer habilidades como la abstracción, la descomposición, el reconocimiento de algoritmos y la automatización con la finalidad de fortalecer las destrezas en el uso de la computación por parte de los estudiantes. Por ejemplo, en el trabajo de da Silva et al. (2020), el PC fue integrado con la finalidad de fortalecer la creación de algoritmos computacionales y programación de computadoras, y se usaron estrategias de evaluación por pares que permitieron que los estudiantes analizaran elementos como la eficiencia de los códigos y algoritmos de sus compañeros. Kaminski y Boscarioli (2018) integraron el PC con un objetivo similar, aunque a través de la creación de objetos de aprendizaje virtuales por parte de los estudiantes.

Por otra parte, la conceptualización del PC como componente de las ciencias de la computación también se evidencia en los estudios revisados, a través de la aplicación de nuevos métodos computacionales en problemas. Por ejemplo, la automatización de soluciones para problemas similares se posiciona como una habilidad fundamental al integrar el PC (de Jesús y Silveira, 2020; Manrique-Losada et al., 2020). En este sentido, para materializar esta habilidad se busca que los estudiantes adopten un rol similar al de un científico de la computación en la forma en que se aproximan a los problemas y crean contenidos computacionales. De esta manera, existen puntos de encuentro entre esta manera de comprender el PC con los planteamientos de Wing (2006), quien propone que una de las características para el desarrollo del PC es pensar como un científico de la computación.

Frente a lo mencionado hasta ahora, se reportan, en la revisión, alternativas como la creación de mapas virtuales a través de dispositivos móviles usando realidad aumentada (Esteves et al., 2019). En este caso, los estudiantes tenían la posibilidad de programar un

Integración del Pensamiento Computacional en la educación primaria y secundaria en Latinoamérica: una revisión sistemática de literatura. D. A. Quiroz-Vallejo, J.A. CarmonaMesa, A. Castrillón-Yepes y J.A. Villa-Ochoa. 
personaje para moverse en un mapa virtual, constituyéndose este como un juguete de programación para desarrollar distintas temáticas en las clases de informática. Por otra parte, von Wangenheim et al. (2019) desarrollaron e implementaron un juego de mesa en el cual es posible integrar, de manera introductoria, conceptos de las ciencias computacionales como los algoritmos y algunos elementos de la programación. Por su parte, Curasma et al. (2019) proponen que el desarrollo de habilidades enfocadas a buscar nuevos métodos computacionales para resolver problemas en la educación primaria y secundaria es una oportunidad que posibilita potenciar el desarrollo económico de la región, dado que con el desarrollo de este tipo de estrategias es posible aumentar la capacidad laboral en TIC y preparar a los estudiantes para la amplia variedad de trabajos del futuro que tendrán conexiones con las ciencias de la computación.

Los estudios revisados también permiten visualizar que el PC, como componente de las ciencias de la computación, fue integrado con la finalidad de reformular problemas para su solución a través de estrategias propias de la computación. Los resultados muestran que, bajo este enfoque, se fomenta el desarrollo de las habilidades de abstracción, planeación, generalización, ejecución y reconocimiento de patrones a través de distintos lenguajes de programación como Scratch (Barbosa y Maltempi, 2019; Basogain et al., 2016). En este sentido, conceptos como variables, asignaciones y operadores relacionales fueron recurrentes para la reformulación de problemas en estos lenguajes.

Además de lo anterior, en múltiples experiencias se apoyó el uso de los lenguajes de programación con otros elementos, como robótica educativa o internet de las cosas. Por ejemplo, Enriquez et al. (2016) implementaron una experiencia con robótica para la enseñanza de la programación en el lenguaje Java y encontraron que este recurso es útil en dos vías: la motivación de los estudiantes y su desempeño en el uso de las estructuras básicas del control de computadoras. Por su parte, Schneider et al. (2020) hicieron uso del internet de las cosas y problemas relacionados con las ciudades inteligentes (Smart Human Cities) para la enseñanza de habilidades de computación y reportaron que esta experiencia promovió un aprendizaje significativo en los estudiantes, quienes pueden enfocarse en una problemática real de su entorno que será importante para su futuro y usar los recursos de la computación para aportar a su solución.

En este apartado se presentó la conceptualización del PC como componente de las ciencias de la computación. Se reconocen dos aproximaciones a esta conceptualización, la primera tiene que ver con la comprensión del PC como una alternativa para el desarrollo de nuevos métodos computacionales para la solución de problemas, en la cual se promueven habilidades como la abstracción, la descomposición de problemas en pequeñas partes, el reconocimiento de algoritmos computacionales y la automatización. En esta aproximación se concibe el PC como un elemento para fortalecer las capacidades de los estudiantes en el uso de la computación. La segunda aproximación es la reformulación de problemas para la aplicación de estrategias computacionales, en la cual los estudiantes toman el rol de creadores de contenidos para emplear sus conocimientos de computación en distintos problemas.

Integración del Pensamiento Computacional en la educación primaria y secundaria en Latinoamérica: una revisión sistemática de literatura. D. A. Quiroz-Vallejo, J.A. CarmonaMesa, A. Castrillón-Yepes y J.A. Villa-Ochoa. 
RED. Revista de Educación a Distancia. Núm. 68, Vol. 21. Artíc. 7, 30-Nov-2021 DOI: http://dx.doi.org/10.6018/red.485321

\section{Reconocimiento de la falta de una conceptualización clara}

En las dos secciones anteriores se presentaron dos tipos de conceptualizaciones del PC que se reflejan de manera explícita o implícita en la literatura revisada. Sin embargo, algunos de los estudios reportaron que, si bien se han desarrollado trabajos importantes que permiten esclarecer conceptualizaciones para integrar el PC en el sistema escolar a nivel internacional (p. ej. Weintrop et al., 2016; Perković et al., 2010), aún es necesario ampliar las discusiones a que se consideren las particularidades de los sistemas educativos latinoamericanos. Por ejemplo, Souza et al. (2019) reconocen el potencial para el desarrollo de procesos interdisciplinares a través del PC como recurso metodológico, sin embargo, informan que las distintas maneras de comprender el PC que conviven en la literatura deben ser matizadas para su debida integración en los sistemas escolares. Mientras tanto, Basogain et al. (2016) ponen en evidencia la necesidad de enriquecer estas conceptualizaciones en la región a partir de la materialización de propuestas que respondan a las necesidades educativas de Latinoamérica.

Aunque el PC es una temática con potencial para aportar a los procesos educativos en Latinoamérica en los intentos por cerrar las brechas entre los países en vías de desarrollo y los desarrollados, y apoyar el fomento de las habilidades necesarias para los trabajos del futuro (Curasma et al. 2019), su integración acarrea retos importantes. En particular, los resultados de esta revisión frente a las conceptualizaciones permiten identificar el desafío de generar consensos frente a las distintas maneras de comprender la temática. En la siguiente sección se describen las estrategias usadas para la integración del PC en los distintos estudios revisados.

\section{Estrategias}

Los resultados de este estudio muestran que la integración del PC en la educación primaria y secundaria se da a través principalmente de la programación en distintos escenarios como la robótica (11), la creación de juegos digitales en lenguajes de programación por bloques (9), la solución de problemas en lenguajes de programación en código (8). Además, en mucha menor medida, se registran también estrategias para la integración del PC desenchufadas (1) a través de experiencias desenchufadas.

\section{Integración del PC a través de la robótica educativa}

En los artículos revisados, la robótica como estrategia fue la más frecuente para la integración del PC. Los dispositivos con los que se trabajó fueron, principalmente, robots basados en placas Arduino y robots Lego Mindstorm NTX. Ejemplo de ello es el estudio de Curasma et al. (2019), quienes a través de robots con placas Arduino Uno, implementaron una experiencia de integración y evaluación del PC con estudiantes de educación media. Estos autores destacan como una característica importante de esta estrategia, el hecho de que los estudiantes pueden probar sus ideas acerca de la interacción de hardware y software rápidamente y ver sus resultados de manera inmediata. Otras experiencias están de acuerdo con estos resultados, como la de Souza et al. (2019), que en sus hallazgos muestra cómo la robótica educativa favorece la percepción de los estudiantes frente a los

Integración del Pensamiento Computacional en la educación primaria y secundaria en Latinoamérica: una revisión sistemática de literatura. D. A. Quiroz-Vallejo, J.A. CarmonaMesa, A. Castrillón-Yepes y J.A. Villa-Ochoa.

Página 17 de 33 
conceptos teóricos de las ciencias de la computación, a través de un enfoque práctico que estimula de manera efectiva las habilidades relacionadas con el PC y las matemáticas.

Estudios como los de García (2015), abordan la robótica educativa y su uso como estrategia para el desarrollo del PC de una manera más amplia, presentando algunos enfoques en los que esta es y ha sido abordada en Uruguay, a partir de la imaginación, el diseño, la construcción y la programación de robots creados por los estudiantes. Sus resultados presentan que esta estrategia sustenta el desarrollo del PC más allá de la programación y trasciende los caminos profesionales a los que los estudiantes se dediquen en el futuro. De este modo, se presenta la codificación como una cuestión que no es exclusivamente de la programación, sino de las diversas formas de comunicación. En este sentido, Rosales et al. (2017) desarrollaron un entorno de programación propio, denominado RITA en RED, con la finalidad de enseñar programación a través de experiencias de combate de robots, su objetivo, además de promover las habilidades técnicas involucradas en la programación de los robots, es el desarrollo de la creatividad, el pensamiento crítico, la comunicación y la colaboración entre pares.

De manera general, los aportes de las estrategias con robótica para la integración del PC reportados en los estudios revisados pueden sintetizarse en tres aspectos: el primero de ellos, son las habilidades específicas del PC a las que estas estrategias aportan, las cuales son la abstracción, la generalización de patrones, la descomposición de problemas y la depuración y detección sistemática de errores; el segundo aporte importante de la robótica educativa para la integración del PC, es la posibilidad que ofrece para la rápida asimilación de conceptos debido a que es una alternativa en la cual los cambios realizados surten efecto de manera inmediata, lo que genera una retroalimentación veloz para los estudiantes; y por último, el tercer aporte, se fundamenta en que el uso de la robótica educativa fomenta la comunicación y colaboración entre pares.

Estos resultados ponen de manifiesto el potencial de la robótica educativa para el desarrollo de habilidades relacionadas con el PC, dado que los estudiantes pueden interactuar tanto con elementos de software como de hardware al tiempo, además de analizar como uno influye en el otro, lo que promueve distintas habilidades del PC como la abstracción o el reconocimiento de patrones. Por otra parte, el uso de la robótica educativa para integrar el PC mostró resultados positivos frente al desarrollo de habilidades comunicativas entre pares y frente a las percepciones de las ciencias de la computación.

\section{Integración del PC a través de la solución de problemas computacionales en lenguajes de programación}

La creación de algoritmos computacionales en diferentes lenguajes para solucionar problemas computacionales se constituyó como una estrategia ampliamente usada para la integración del PC en la educación primaria y secundaria. En esta estrategia, las experiencias tuvieron en cuenta distintas maneras de trabajar con los algoritmos, tales como la programación entre pares o la programación basada en desafíos; además, los lenguajes usados tienden a ser en líneas de código, en comparación a la estrategia de

Integración del Pensamiento Computacional en la educación primaria y secundaria en Latinoamérica: una revisión sistemática de literatura. D. A. Quiroz-Vallejo, J.A. CarmonaMesa, A. Castrillón-Yepes y J.A. Villa-Ochoa. 
robótica educativa, en la cual se usaron mayoritariamente lenguajes de programación con bloques.

La programación entre pares fue una estrategia usada en el trabajo de da Silva et al. (2020), en la cual el estudiante escribe el código y el instructor se sitúa a su lado para supervisar, ambos intercambiaban impresiones y discutían al respecto de la eficiencia de las líneas escritas y la posibilidad de mejorarlas. Por su parte, González et al. (2018) diseñaron y ejecutaron estrategias para la enseñanza del lenguaje $\mathrm{C}++$ a través del fomento de habilidades como el pensamiento sistemático y la abstracción; en esta investigación los estudiantes recibían una parte de la clase con los conceptos básicos de programación y el resto se dedicaba a explorar y trabajar en solucionar situaciones propuestas por los instructores, quienes hacían sesiones de retroalimentación con programación entre pares cuando les era solicitado. Por otra parte, en una de las experiencias revisadas se usó Screen Turtle (Araujo et al., 2018), un ambiente de aprendizaje inspirado en el trabajo de Seymour Papert para introducir a los estudiantes en la programación a través de la creación de figuras geométricas con comandos simples y conceptos de programación como bucles, funciones, estructuras y parámetros; los resultados de este estudio muestran que este tipo de programación con comandos simples tuvo un impacto positivo en la motivación de los estudiantes.

Los resultados relacionados con la integración del PC, a través de la programación, enseñan el potencial del trabajo en equipo en estos procesos, que a priori parecen actividades pensadas para realizarse de forma individual únicamente; además, se muestra el potencial de lenguajes de programación de bajo nivel de complejidad para introducir a los estudiantes en las habilidades relacionadas con el PC tanto en las ciencias de la computación como en áreas diferentes del sistema escolar. En este sentido, los aportes de la integración del PC a través de la solución de problemas computacionales con lenguajes de programación se sintetizan en dos aspectos fundamentales: el primero, relacionado con las habilidades del PC que permiten desarrollar el dominio de lenguajes de programación, como la abstracción y los procesos de pensamiento sistémico, en los cuáles el rol de la programación por pares fue fundamental para hacer mejoras continuas a las soluciones de los equipos de estudiantes. Por otra parte, el segundo aspecto tiene que ver con los elementos básicos de programación como los bucles, funciones, estructuras y parámetros, cuyo aprendizaje es potenciado a través de lenguajes con comandos simples y aportan a la motivación de los estudiantes frente al proceso de solucionar los problemas propuestos.

\section{Integración del PC a través de juegos digitales}

La creación de juegos digitales a través de lenguajes de programación por bloques fue otra de las estrategias usadas en múltiples de las experiencias revisadas en este estudio. Scratch fue el recurso más utilizado para la creación de contenidos, dado que posibilita el desarrollo de animaciones, juegos interactivos, entre otros elementos. En particular, es destacable el trabajo de Kaminski y Boscarioli (2018), en el cual los estudiantes de educación primaria utilizan Scratch para apoyar los procesos educativos de sus compañeros en niveles más tempranos de escolaridad; los resultados de este estudio mostraron que esta estrategia permite desarrollar tanto habilidades del PC como procesos

Integración del Pensamiento Computacional en la educación primaria y secundaria en Latinoamérica: una revisión sistemática de literatura. D. A. Quiroz-Vallejo, J.A. CarmonaMesa, A. Castrillón-Yepes y J.A. Villa-Ochoa. 
interdisciplinares entre la programación y las disciplinas que los estudiantes buscaban enseñar a sus pares. En estos procesos existe un alto grado de apropiación por parte de los estudiantes que tienen el rol de creadores de contenido educativo. Por otra parte, von Wangenheim et al. (2017) también usaron Scratch para desarrollar juegos digitales con finalidad educativa relacionados con la historia, sus resultados muestran que los juegos ayudan a que los estudiantes aumenten su nivel de compromiso con la disciplina de humanidades, además de aumentar los niveles de trabajo en equipo de los estudiantes a través de la programación del juego entre pares.

Para la integración del PC en las clases de matemáticas, estudios como los de Barbosa y Maltempi (2019) mostraron el desarrollo de un juego en Scratch para reforzar conceptos básicos de ecuaciones de primer grado; entre los resultados de la aplicación de esta estrategia, además de la comprensión de los conceptos matemáticos, tienen que ver con la adquisición de habilidades de abstracción, automatización, simulación y generalización, propias del PC. En la experiencia reportada por da Silva et al. (2020), los estudiantes también desarrollaron un juego en Scratch, en el cual fue necesario poner en situación conceptos matemáticos como la función cuadrática para lograr construir el algoritmo de movimiento de los personajes del juego. Estos resultados muestran que en la integración del PC a través de estrategias como el desarrollo de juegos digitales es posible encontrar situaciones en las cuales los diferentes conocimientos disciplinares cobran sentido y pueden integrarse con elementos de las tecnologías digitales y las habilidades que promueve el PC.

Los juegos digitales como estrategia para la integración del PC en la educación primaria y secundaria se caracterizaron por ser construidos en el lenguaje de programación por bloques Scratch, el cual ha sido ampliamente usado a nivel internacional por su característica de ser fácil de aprender a usar, pero sin limitar el tipo de creaciones que de allí pueden derivar. Es decir, Scratch es un lenguaje de programación fácil de aprender, pero con un alto potencial para complejizarse en la medida en que los estudiantes adquieren nuevas habilidades. Bajo estas condiciones, los estudios revisados permiten reconocer el desarrollo de habilidades del PC como la automatización y la simulación de procesos. Además, algunos de los estudios que usaron esta estrategia (p. ej. Kaminski y Boscarioli, 2018; von Wangenheim et al., 2019) reportan que la creación de juegos digitales posibilita desarrollar procesos interdisciplinarios dado que la historia de estos se puede desarrollar en función de distintas temáticas.

\section{Integración del PC a través de actividades desenchufadas}

EI PC desenchufado refiere a actividades que tienen el potencial para fomentar habilidades del PC sin incluir el uso de ordenadores o dispositivos electrónicos, y aunque en la literatura se reporta su pertinencia e importancia (Zapata-Ros, 2019), en los resultados de este estudio solo se registra una experiencia en la cual se usa esta estrategia para integrar el PC en la educación media. El estudio denomina la estrategia como Splashcode, un juego de mesa que tiene por objetivo apoyar los procesos de comprensión de los algoritmos (von Wangenheim et al., 2019); sus resultados mostraron que el juego tiene un impacto positivo tanto en la motivación por aprender conceptos y habilidades relacionadas

Integración del Pensamiento Computacional en la educación primaria y secundaria en Latinoamérica: una revisión sistemática de literatura. D. A. Quiroz-Vallejo, J.A. CarmonaMesa, A. Castrillón-Yepes y J.A. Villa-Ochoa.

Página 20 de 33 
con el PC como en cuestiones concretas, tales como la descomposición de los problemas, el reconocimiento del concepto de algoritmo y sus usos para completar tareas. De acuerdo con estos resultados, las comunidades académicas y educativas en Latinoamérica tienen la oportunidad de construir nuevas actividades de PC desenchufado que posibiliten la integración del PC en escenarios donde no hay acceso estable a internet ni a dispositivos electrónicos.

Finalmente, se identifica una necesidad importante en la región frente a la creación de estrategias, ya sean basadas en la programación en sus distintos tipos o desenchufadas, que permitan evaluar los aprendizajes de los estudiantes y el desarrollo de sus habilidades del PC. Esto sugiere la necesidad de enriquecer la literatura en la región sobre estrategias evaluativas adecuadas para cada uno de los niveles educativos con la finalidad de generar recursos tanto para profesores como investigadores que permitan rastrear una trayectoria completa del desarrollo del PC en los estudiantes a lo largo de sus vidas académicas.

\section{Discusión}

En la literatura internacional se identifican múltiples revisiones que aportan de manera directa a las conceptualizaciones y estrategias para la implementación del PC en la educación primaria y secundaria. Por ejemplo, Shute et al. (2017) describen el PC como una base conceptual necesaria para resolver problemas de manera eficaz y eficiente con soluciones que pueden ser reutilizadas en diferentes contextos. Del mismo modo, Barr y Stephenson (2011) mencionan que el PC es una aproximación a la solución de problemas de una manera en la que esta puede ser implementada en computadoras y pone a los estudiantes en un rol de constructores de recursos. Estas conceptualizaciones se han constituido como herramientas útiles para la integración del PC en las clases de ciencias de la computación o informática en países como Estados Unidos o los miembros de la Unión Europea.

Existen estudios, especialmente revisiones de literatura, a nivel internacional que se han preocupado por la integración del PC a través de disciplinas diferentes a las ciencias de la computación. Al respecto, trabajos como los de Pérez (2018) y Weintrop et al. (2016) reportan de manera especial asuntos particulares para tener en cuenta en la integración del PC en las clases de matemáticas y ciencias. Mientras tanto, Perković et al. (2010) desarrollaron un marco de trabajo para el PC a través de disciplinas como las artes y la literatura. En Latinoamérica, la integración del PC en la educación primaria y secundaria está consolidándose como una temática de amplio interés para investigadores y profesores, por lo que las conceptualizaciones y estrategias concretas para ser integrado a la realidad de la región, tienen un papel cada vez más relevante.

La mayoría de las experiencias reportadas en esta revisión se enmarcan en las áreas de ciencias de la computación o informática y hacen parte, principalmente, de investigaciones de corto alcance o pruebas piloto para el fomento de la temática. En otras palabras, en la mayoría de las experiencias revisadas en este estudio, el PC aún no se constituye como un elemento incluido de manera formal en los currículos escolares. Con relación al predominio de las ciencias de la computación y la informática, estos resultados ratifican la revisión de

Integración del Pensamiento Computacional en la educación primaria y secundaria en Latinoamérica: una revisión sistemática de literatura. D. A. Quiroz-Vallejo, J.A. CarmonaMesa, A. Castrillón-Yepes y J.A. Villa-Ochoa. 
Shute et al. (2017). Sin embargo, la aún incipiente inserción en los currículos se constituye como una particularidad de la región, dado que, en países como Estados Unidos y los miembros de la unión europea, el PC se constituye en la actualidad como una parte importante de los currículos escolares (Bocconi et al., 2016).

Los argumentos anteriores permiten delimitar dos oportunidades para el fortalecimiento de la temática en la región: primero, se identifica un amplio potencial de investigación frente a experiencias para el desarrollo del PC en disciplinas distintas a las ciencias de la computación para establecer cuáles son las posibilidades y límites de esta temática a través de distintas áreas del conocimiento (Grover y Pea, 2013; Villa-Ochoa y Castrillón-Yepes, 2020). La segunda oportunidad para fortalecer la integración del PC en Latinoamérica tiene que ver con la implementación de experiencias de largo aliento que ofrezcan un análisis profundo acerca del impacto de la integración del PC en la educación primaria y secundaria (Quiroz-Vallejo, 2020). Estos análisis de mayor profundidad tendrán el potencial de aportar para los diseños curriculares en los que se integre de manera formal el PC en las aulas de la región (Curasma y Curasma, 2020).

Frente al potencial para el desarrollo e integración del PC en disciplinas distintas a las ciencias de la computación se deriva el desafío de trascender su naturaleza de vehículo para alcanzar objetivos propios de las temáticas en las que se integra. En este sentido, la comprensión de la actividad de codificación emerge como fundamental, en tanto su naturaleza va más allá de escribir programas computacionales para solucionar problemas disciplinares específicos. Al contrario, la actividad de codificar es susceptible de entenderse desde el proceso de desarrollar cálculos matemáticos hasta la previsión de las consecuencias que acciones específicas pueden tener sobre un sistema (Coll, 2018). Bajo esta mirada, el PC, además de recurso metodológico, permite que los estudiantes desarrollen capacidades y actitudes derivadas de las ciencias de la computación, pero adaptadas a enfrentar problemas más allá de la codificación o la construcción de algoritmos. Por consiguiente, más allá que el análisis de los programas construidos por los estudiantes toma relevancia el análisis del proceso de pensamiento llevado a cabo y el manejo de las herramientas cognitivas y metacognitivas para su construcción (Zapata-Ros, 2018).

En Latinoamérica, las conceptualizaciones evidenciadas en la integración del PC en la educación primaria y secundaria son aún tema de discusión y no hay un consenso al respecto entre la comunidad académica de la región (Souza et al., 2019; Basogain et al., 2016); y, en particular, se reconocen brechas en la integración tanto en las asignaturas de informática/tecnología como en las distintas disciplinas. En el caso de informática/tecnología, aunque estas disciplinas son en las cuáles más se ha trabajado la temática del PC y a partir de las cuales se ha desplegado a otros campos de conocimiento, existen críticas frente a su dimensión de reformulación de problemas (Cansu y Cansu, 2019). Por ejemplo, Hemmendinger (2010) argumenta que la reformulación de problemas difíciles es un elemento típico de todos los dominios de solución de problemas complejos, la filosofía ha pensado de manera recursiva antes y en las matemáticas ya integran elementos de abstracción, al igual que todas las ciencias que usan modelos. Estas críticas ponen de manifiesto una necesidad de elementos identitarios propios de la temática que le brinden una esencia clara a la comunidad académica del PC. Al respecto, esta revisión

Integración del Pensamiento Computacional en la educación primaria y secundaria en Latinoamérica: una revisión sistemática de literatura. D. A. Quiroz-Vallejo, J.A. CarmonaMesa, A. Castrillón-Yepes y J.A. Villa-Ochoa.

Página 22 de 33 
muestra que posiblemente el factor diferenciador del PC para la educación primaria y secundaria tenga que ver con la inclusión al mismo tiempo de los distintos elementos que componen el PC y la conexión que este tiene con las tecnologías digitales y las necesidades de formación para los mercados laborales futuros (Tabesh, 2017).

Por otra parte, frente a la conceptualización del PC a través de las diferentes disciplinas que componen los currículos escolares, se presentan puntos que favorecen su integración, como sus posibles conexiones con las matemáticas en aspectos como la modelación, solución de problemas, análisis de datos y estadística (Sneider et al., 2014). Del mismo modo, se encuentran similitudes con las prácticas de datos y de pensamiento sistémico propuestos por Weintrop et al. (2016) para la integración del PC en las clases de matemáticas y ciencias. Con este contexto, las matemáticas y las ciencias de la computación se constituyeron en esta revisión como las dos disciplinas con mayor evidencia de integración interdisciplinar, en la cual los estudiantes construyen conexiones entre estas dos para resolver problemas complejos y se diferencia de manera clara el aprendizaje en cada una (Gao et al., 2020). Sin embargo, en las experiencias que integraron el PC en disciplinas como las ciencias sociales o las humanidades la tendencia fue marcada hacia el uso del PC como un vehículo para lograr aprendizajes específicos en estas disciplinas, dejando de lado elementos clave de las ciencias de la computación con potencial de ser integrados.

De acuerdo con lo mencionado, se reconoce la necesidad de ampliar en investigaciones y experiencias educativas en la región que integren el PC y disciplinas como las ciencias naturales, las humanidades y las ciencias sociales, dado que la cantidad de documentos revisados fue baja, además de identificarse en ellos limitaciones para desarrollar conexiones interdisciplinares. Lo anterior está en consonancia con las problemáticas que históricamente ha tenido la región frente a la integración de tecnologías digitales en los sistemas educativos, dado que este proceso, aunque se ha caracterizado por la preocupación latente por cerrar las brechas de equipamiento tecnológico, ha encontrado problemas en la alfabetización digital de profesores y estudiantes, lo que conlleva a un uso instrumental de la tecnología (Artopoulos y Kozak, 2012) y, por consiguiente, a una baja integración disciplinar en las clases de informática y tecnología. En la Figura 3. se resumen las conceptualizaciones del PC identificadas en la revisión.

\section{Figura 3.}

Conceptualizaciones del PC identificadas en la revisión 


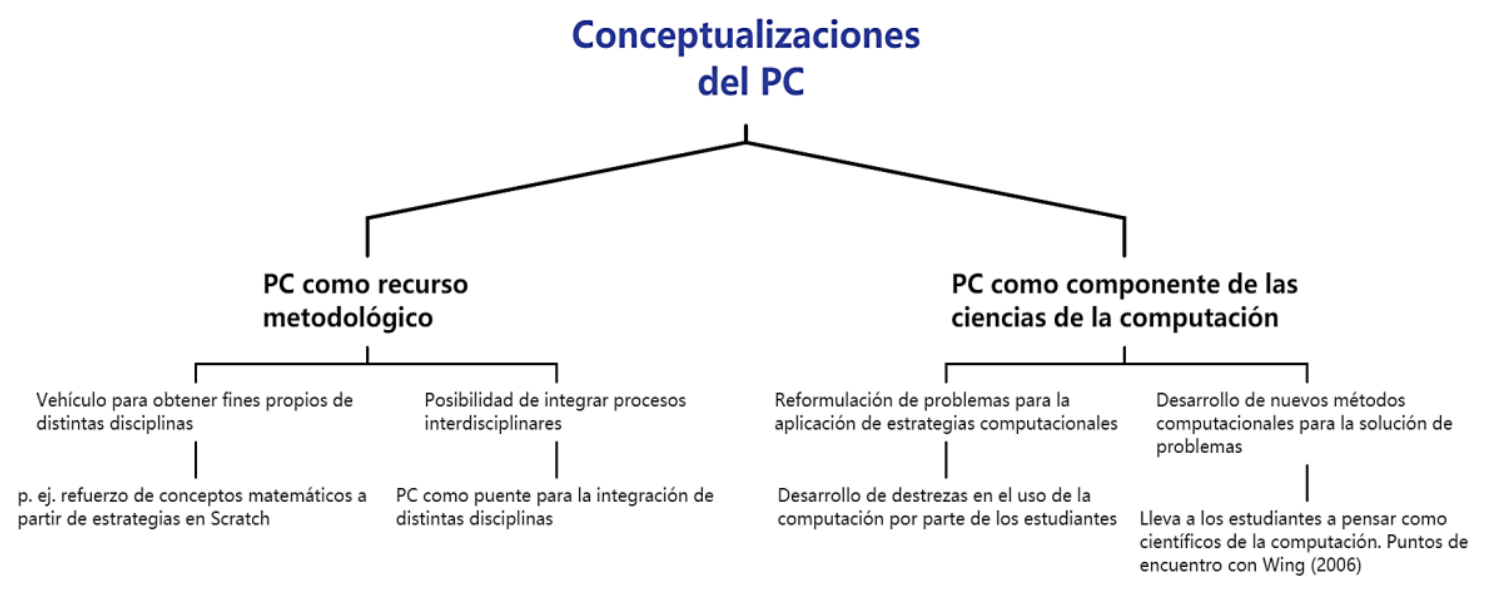

Elaboración de los autores

Es posible ver que algunas de las conceptualizaciones del PC que se presentan en los trabajos están en correspondencia con las definiciones del PC para las clases de matemáticas y ciencias en la educación primaria y secundaria que proponen Weintrop et al. (2016); estos autores proponen una serie de prácticas relacionadas con el PC, entre ellas están las prácticas de solución de problemas computacionales y la del pensamiento sistémico. En particular, en las experiencias que fueron categorizadas en la integración de PC como recurso metodológico, se enmarcan en las prácticas de PC como proceso sistémico, dado que, en general, sus objetivos apuntan a la solución de problemas a través de la comprensión de las relaciones entre las características del problema, las posibles soluciones y la investigación de este como un sistema complejo (Souza et al., 2019; Rodrigues et al., 2016). Por otra parte, las experiencias categorizadas en la integración del PC como objeto de estudio se enmarcan en la práctica de solución de problemas computacionales, dado que entre sus características fue identificada la reformulación de problemas para ser solucionados con estrategias computacionales y el uso directo de la programación (Scheneider et al., 2020; Enriquez et al., 2016).

Mientras los trabajos revisados presentan puntos de encuentro con algunas de las tendencias internacionales para la integración del PC, en la misma medida hay otros marcos que se dejan de lado y que presentan una oportunidad para ampliar en futuras investigaciones. Por ejemplo, en el caso de las matemáticas, el PC puede explorarse como un elemento para aportar en la tolerancia por la ambigüedad, como lo expresa Pérez (2018) en su marco de trabajo para el PC y las matemáticas. La ambigüedad aporta a los estudiantes en las clases de matemáticas, en tanto les brinda la posibilidad de clarificar por sus medios qué es lo que saben y qué es lo que no saben, pueden considerar múltiples aproximaciones a las situaciones y mejorar sus habilidades de trabajo colaborativo. En el caso de las ciencias naturales, se identifica como una oportunidad para ampliar en futuras investigaciones la manera en que el PC puede afectar la forma como los estudiantes entienden las ciencias en general y la experimentación en particular (Denning, 2017). Por otra parte, es necesario identificar aproximaciones para la integración del PC en disciplinas

Integración del Pensamiento Computacional en la educación primaria y secundaria en Latinoamérica: una revisión sistemática de literatura. D. A. Quiroz-Vallejo, J.A. CarmonaMesa, A. Castrillón-Yepes y J.A. Villa-Ochoa. 
RED. Revista de Educación a Distancia. Núm. 68, Vol. 21. Artíc. 7, 30-Nov-2021

DOI: http://dx.doi.org/10.6018/red.485321

diferentes a las ciencias de la computación, las ciencias naturales y las matemáticas, dado que son muy escasos los estudios que integran el PC en estas áreas en la región.

En los resultados de esta revisión, frente a las estrategias usadas para la integración del PC, se encontró que la robótica educativa se constituye como la estrategia más usada en las experiencias analizadas. El uso de estrategias relacionadas con la robótica se ha reportado como con amplio potencial, dado que posibilita que los estudiantes tengan experiencias tangibles (Shute et al., 2017); en concordancia, el argumento principal por el cual se integró la robótica en las experiencias revisadas es la interacción inmediata entre software y hardware. Estos hallazgos contrastan con revisiones internacionales como la de Hsu et al. (2017), en la cual este tipo de estrategias solo fueron usadas de una forma minoritaria en comparación. De este modo, aunque la robótica educativa se consolida en Latinoamérica como una estrategia en la cual vale la pena ahondar frente a sus potencialidades para la integración del PC en la educación primaria y secundaria, también es necesario reconocer las limitaciones de recursos e infraestructura en la región, que podrían limitar la integración del PC bajo esta modalidad.

Por otra parte, el uso de distintos lenguajes de programación, tanto para la solución de problemas en las diferentes disciplinas, como para la solución de problemas computacionales y la creación de juegos digitales, continúa consolidándose como una de las estrategias más usadas para la integración del PC en todos los niveles educativos. En este sentido, se observa que las necesidades de formación en programación se constituyen como una cuestión fundamental para integrar el PC en la educación primaria y secundaria. Estos resultados están en coherencia con Villa-Ochoa y Castrillón-Yepes (2020), quienes encontraron la misma tendencia en la educación superior. Además, el uso de lenguajes de programación por bloques es el más frecuente; en especial Scratch, el cual es más usado que otros softwares similares dado que es un recurso versátil que posibilita desarrollar distintos tipos de programas como juegos, storytelling, entre otros. Más aún, Scratch ha sido objeto de estudio de manera tanto teórica (Brennan y Resnick, 2012) como empírica (Zhang y Nouri, 2019), de modo que su uso para la integración del PC ha sido ampliamente validado en la comunidad académica.

Es importante resaltar que el uso de estrategias para la integración del PC desenchufadas brilló por su ausencia en la revisión. Solo un estudio, desarrollado por von Wangenheim et al. (2019), desarrolló una estrategia en esta categoría. En este sentido, se identifica una oportunidad para ampliar en investigaciones que reporten distintos recursos para la integración del PC sin el uso de dispositivos electrónicos, necesidad que se recrudece al considerar que en Latinoamérica aún hay regiones que no tienen acceso ni a computadoras ni a internet de manera constante. Finalmente, se resalta una necesidad en la región por generar distintos recursos que permitan evaluar tanto los aprendizajes en función de la computación por parte los estudiantes, como los aprendizajes favorecidos por el PC en otras disciplinas. Esta necesidad se corresponde con los resultados presentados por Tang et al. (2020), quienes reportan la importancia de construir estrategias evaluativas que se alineen con las distintas conceptualizaciones existentes del PC.

Integración del Pensamiento Computacional en la educación primaria y secundaria en Latinoamérica: una revisión sistemática de literatura. D. A. Quiroz-Vallejo, J.A. CarmonaMesa, A. Castrillón-Yepes y J.A. Villa-Ochoa.

Página 25 de 33 


\section{Conclusiones}

El objetivo de esta revisión fue analizar la integración del PC en la educación primaria y secundaria en Latinoamérica. Para ello, se tuvo en cuenta dos categorías: conceptualizaciones del PC y estrategias para su integración. Los resultados de este estudio aportan a las discusiones frente al PC en educación al identificar mayoritariamente estrategias que buscaban explorar la integración del PC en períodos muy cortos. En este sentido, hay una necesidad latente de ampliar investigaciones de mayor duración que posibiliten generar análisis profundos sobre los aportes y desafíos de integrar el PC en los currículos escolares a largo plazo, tanto en las ciencias de la computación y la informática como a través de las otras áreas de los currículos.

Las discusiones frente a las conceptualizaciones del PC para la educación primaria y secundaria deben ampliarse en la región. Aunque en la actualidad se evidencia que las tendencias hacia las que se dirigen las investigaciones están en concordancia con algunas investigaciones a nivel internacional de largo alcance, es necesario ampliar la perspectiva de la temática en la región desde el punto de vista de las problemáticas puntuales que se manifiestan en la región, como el acceso a internet e infraestructura de tecnologías digitales. En este sentido, aunque se han explorado distintas estrategias para la integración del PC a través de dispositivos electrónicos, el PC desenchufado solo se evidenció en uno de los estudios revisados. Es necesario explorar esta modalidad del PC en la región porque permite ampliar la cobertura para su integración en los currículos en regiones en las cuales aún no existe conectividad constante a internet o acceso a dispositivos como celulares y computadoras. Por último, se deben tener en cuenta para futuras investigaciones relacionadas con el PC, la ampliación acerca de las implicaciones de la integración del PC como recurso metodológico en distintas disciplinas y las implicaciones de su uso a largo plazo.

\section{Agradecimientos}

Se agradece a Siemens Stiftung y Siemens Caring Hands por el financiamiento del proyecto "Fomento de la educación STEAM a partir del pensamiento computacional", el cual está enmarcado en la iniciativa Educación STEM para la innovación en América Latina.

Presentación del artículo: 29 de junio de 2021

Fecha de aprobación: 3 de septiembre de 2021

Fecha de publicación: 30 de noviembre de 2021

Quiroz-Vallejo, D.A., Carmona-Mesa, J.A., Castrillón-Yepes, A., \& Villa-Ochoa, J.A. (2021). Integración del Pensamiento Computacional en la educación primaria y secundaria en Latinoamérica: una revisión sistemática de literatura. RED. Revista de educación a distancia, 21(68). http://dx.doi.org/10.6018/red.485321

Integración del Pensamiento Computacional en la educación primaria y secundaria en Latinoamérica: una revisión sistemática de literatura. D. A. Quiroz-Vallejo, J.A. CarmonaMesa, A. Castrillón-Yepes y J.A. Villa-Ochoa. 
RED. Revista de Educación a Distancia. Núm. 68, Vol. 21. Artíc. 7, 30-Nov-2021

DOI: http://dx.doi.org/10.6018/red.485321

\section{Financiación:}

El presente trabajo se inserta en el proyecto de investigación denominado "Fomento de la educación STEAM a partir del pensamiento computacional” (2021-39890)

\section{Referencias}

Araujo, L. G. J., Bittencourt, R. A., \& Santos, D. M. (2018, February). An analysis of a mediabased approach to teach programming to middle school students. In Proceedings of the 49th ACM Technical Symposium on Computer Science Education (pp. 1005-1010). https://doi.org/10.1145/3159450.3159526

Artopoulos, A., \& Kozak, D. (2012). Topografías de la integración de Tic en Latinoamérica: Hacia la interpretación de los estilos de adopción de tecnología en educación. En D. Goldin, M. Kriscautzky \& F. Perelman (Eds) Las TIC en la escuela, nuevas herramientas para viejos y nuevos problemas (pp. 393-452). Océano.

Azevedo, G. T. D., \& Maltempi, M. V. (2020). Processo de Aprendizagem de Matemática à luz das Metodologias Ativas e do Pensamento Computacional. Ciência \& Educação (Bauru), 26. https://doi.org/10.1590/1516-731320200061

Barbosa, L. L., \& Maltempi, M. V. (2019). Recognizing possibilities of computational tinking when teaching first-degree equations. ACM International Conference Proceeding Series, 57-64. https://doi.org/10.1145/3311/0890.3311898

Barr, V., \& Stephenson, C. (2011). Bringing computational thinking to K-12: what is Involved and what is the role of the computer science education community?. Acm Inroads, 2(1), 48-54. https://doi.org/10.1145/1929887.1929905

Basogain, X., \& Olmedo, M. E. (2020). Integración de Pensamiento Computacional en Educación Básica. Dos Experiencias Pedagógicas de Aprendizaje Colaborativo online. Revista de Educación a Distancia (RED), 20(63). https://doi.org/10.6018/red.409481

Basogain, X., Olabe, M. A., Olabe, J. C., Ramírez, R., Del Rosario, M., \& Garcia, J. (2016, September). PC-01: Introduction to computational thinking: Educational technology in primary and secondary education. In 2016 International Symposium on Computers in Education (SIIE) (pp. 1-5). IEEE. https://doi.org/10.1109/SIIE.2016.7751816

Bocconi, S., Chioccariello, A., Dettori, G., Ferrari, A., Engelhardt, K., Kampylis, P., \& Punie, Y. (2016). Developing computational thinking in compulsory education. European Commission, JRC Science for Policy Report, 68. https://doi.org/10.2791/792158

Brennan, K., \& Resnick, M. (2012, April). New frameworks for studying and assessing the development of computational thinking. In Proceedings of the 2012 annual meeting of the American educational research association, Vancouver, Canada (Vol. 1, p. 25).

Integración del Pensamiento Computacional en la educación primaria y secundaria en Latinoamérica: una revisión sistemática de literatura. D. A. Quiroz-Vallejo, J.A. CarmonaMesa, A. Castrillón-Yepes y J.A. Villa-Ochoa. 
RED. Revista de Educación a Distancia. Núm. 68, Vol. 21. Artíc. 7, 30-Nov-2021

DOI: http://dx.doi.org/10.6018/red.485321

Cansu, S. K., \& Cansu, F. K. (2019). An Overview of Computational Thinking. International Journal of Computer Science Education in Schools, 3(1), n1. https://doi.org/10.21585/ijcses.v3i1.53

Carmona-Mesa, J. A., Krugel, J., \& Villa-Ochoa, J. A. (2021). La formación de futuros profesores en tecnología. Aportes al debate actual sobre los Programas de Licenciatura en Colombia. En A. Richit \& H. Oliveira (Eds.), Formação de professores em contextos permeados pelas tecnologias digitais. Brazil: Livraria da Física

Carmona-Mesa J.A., Quiroz-Vallejo D.A., Villa-Ochoa J.A. (2022) Disciplinary Knowledge of Mathematics and Science Teachers on the Designing and Understanding of Robot Programming Algorithms. In: Merdan M., Lepuschitz W., Koppensteiner G., Balogh R., Obdržálek D. (eds) Robotics in Education. Advances in Intelligent Systems and Computing, vol 1359. Springer, Cham. https://doi.org/10.1007/978-3-030-82544-7_16

Coll, C.(2018). Reseña del libro "El pensamiento computacional. Análisis de una competencia clave". RED Revista de Educación a Distancia, Reseñas. http://dx.doi.org/10.6018/red/resenas/01

Corzo, S. M., Mantilla, O., \& Quintero Rojas, J. E. (2020). Transformando proceso de enseñanza-aprendizaje con la implementación de un aula virtual siguiendo el modelo STEAM. In Tecnologías educativas y estrategias didácticas (pp. 743-758). Servicio de Publicaciones Universidad de Málaga

Costa, E. J. F., Campos, L. M. R. S., \& Guerrero, D. D. S. (2017, October). Computational thinking in mathematics education: A joint approach to encourage problem-solving ability. In 2017 IEEE Frontiers in Education Conference (FIE) (pp. 1-8). IEEE. https://doi.org/10.1109/FIE.2017.8190655

Curasma, R. P., Jara, N. J., Curasma, H. P., \& Ornetta, V. C. (2019, August). Assessment of Computational Thinking in regular basic education: case IETP "José Obrero". In 2019 IEEE XXVI International Conference on Electronics, Electrical Engineering and Computing (INTERCON) (pp. https://doi.org/10.1109/INTERCON.2019.8853613

Curasma, R., \& Curasma, H. (2020). Computational thinking in school education in South America: Systematic review of the literature. Proceedings of the 2020 IEEE 27th International Conference on Electronics, Electrical Engineering and Computing, INTERCON 2020, 9-12. https://doi.org/10.1109/INTERCON50315.2020.9220200

da Silva, F. F., Aylon, L. B. R., \& Flôr, D. E. (2020, October). Teaching Computational Thinking to a Student with Attention Deficit Through Programming. In 2020 IEEE Frontiers in Education Conference (FIE) (pp. 1-9). IEEE. https://doi.org/10.1109/FIE44824.2020.9273915

Integración del Pensamiento Computacional en la educación primaria y secundaria en Latinoamérica: una revisión sistemática de literatura. D. A. Quiroz-Vallejo, J.A. CarmonaMesa, A. Castrillón-Yepes y J.A. Villa-Ochoa. 
RED. Revista de Educación a Distancia. Núm. 68, Vol. 21. Artíc. 7, 30-Nov-2021 DOI: http://dx.doi.org/10.6018/red.485321

de Jesus Â.M., Silveira I.F. (2020) Applying Agile Software Engineering to Develop Computational Thinking in Children. In: Rocha Á., Adeli H., Reis L., Costanzo S., Orovic I., Moreira F. (eds) Trends and Innovations in Information Systems and Technologies. WorldCIST 2020. Advances in Intelligent Systems and Computing, vol 1159. Springer, Cham. https://doi.org/10.1007/978-3-030-45688-7_74

de Souza, C. S., Salgado, L. C., Leitão, C. F., \& Serra, M. M. (2014, June). Cultural appropriation of computational thinking acquisition research: seeding fields of diversity. In Proceedings of the 2014 conference on Innovation \& technology in computer science education (pp. 117-122). https://doi.org/10.1145/2591708.2591729

Denning, P. J. (2009). The profession of IT Beyond computational thinking. Communications of the ACM, 52(6), 28-30. https://doi.org/10.1145/1516046.1516054

Denning, P. J. (2017). Computational thinking in science. American Scientist, 105(1), 13-17.

Enriquez, C., Aguilar, O., \& Domínguez, F. (2016). Using robot to motivate computational thinking in high school students. IEEE Latin America Transactions, 14(11), 4620-4625. https://doi.org/10.1109/TLA.2016.7795838

Esteves, A., Santana, A., \& Lyra, R. (2019). Use of Augmented Reality for Computational Thinking Stimulation through Virtual Toys. In Anais do XXI Simpósio de Realidade Virtual e Aumentada, (pp. 252-256). Porto Alegre: SBC https://doi.org/10.1109/SVR.2019.00031

Fessakis, G., Komis, V., Mavroudi, E., \& Prantsoudi, S. (2018). Exploring the scope and the conceptualization of computational thinking at the K-12 classroom level curriculum. In Computational Thinking in the STEM Disciplines (pp. 181-212). Springer, Cham. https://doi.org/10.1007/978-3-319-93566-9_10

García, J. M. (2015). Robótica Educativa. La programación como parte de un proceso educativo. Revista de Educación a Distancia (RED), (46). Recuperado de https://revistas.um.es/red/article/view/240201

Gao, X., Li, P., Shen, J., \& Sun, H. (2020). Reviewing assessment of student learning in interdisciplinary STEM education. International Journal of STEM Education, 7(24), 1-14. https://doi.org/10.1186/s40594-020-00225-4

González, F., López, C., \& Castro, C. (2018, November). Development of Computational Thinking in High School Students: A Case Study in Chile. In 2018 37th International Conference of the Chilean Computer Science Society (SCCC) (pp. 1-8). IEEE. https://doi.org/10.1109/SCCC.2018.8705239

Grover, S., \& Pea, R. (2013). Computational Thinking in K-12: A Review of the State of the Field. Educational Researcher, 42(1), 38-43. https://doi.org/10.3102/0013189X12463051

Integración del Pensamiento Computacional en la educación primaria y secundaria en Latinoamérica: una revisión sistemática de literatura. D. A. Quiroz-Vallejo, J.A. CarmonaMesa, A. Castrillón-Yepes y J.A. Villa-Ochoa. 
RED. Revista de Educación a Distancia. Núm. 68, Vol. 21. Artíc. 7, 30-Nov-2021 DOI: http://dx.doi.org/10.6018/red.485321

Grover, S., \& Pea, R. (2018). Computational thinking: A competency whose time has come. En S. Sentances, E. Barendsen, \& C. Schulte (Eds.), Computer science education: Perspectives on teaching and learning in school (Bloomsbury, pp. 20-35). Londres

Hemmendinger, D. (2010). A plea for modesty. Acm Inroads, 1(2), 4-7. https://doi.org/10.1145/1805724.1805725

Hsu, T.-C., Chang, S.-C., \& Hung, Y.-T. (2018). How to learn and how to teach computational thinking: Suggestions based on a review of the literature. Computers \& Education, 126(September 2017), 296-310. https://doi.org/10.1016/j.compedu.2018.07.004

Huerta, C. S., \& Velázquez, M. (2021). Pensamiento computacional como una habilidad genérica: una revisión sistemática. Ciencia Latina, 5(1), 1055-1078. https://doi.org/10.37811/cl_rcm.v5i1.311

Jara, I., \& Hepp, P. (2016). Enseñar Ciencias de la Computación: Creando oportunidades para los jóvenes de América Latina. Recuperado de https://www.researchgate.net/publication/309761093_Ensenar_Ciencias_de_la_Compu tacion_Creando_oportunidades_para_los_jovenes_de_America_Latina

Jara, I., \& Hepp, P. (2016). Enseñar Ciencias de la Computación: Creando oportunidades para los jóvenes de América Latina. Recuperado de http://repositorio.minedu.gob.pe/handle/20.500.12799/5936

Kale, U., Akcaoglu, M., Cullen, T., Goh, D., Devine, L., Calvert, N., \& Grise, K. (2018). Computational what? Relating computational thinking to teaching. TechTrends, 62(6), 574-584. https://doi.org/10.1007/s11528-018-0290-9

Kaminski, M. R., \& Boscarioli, C. (2018, October). Production of scratch learning objects by elementary school students. In 2018 XIII Latin American Conference on Learning Technologies (LACLO) (pp. 299-306). IEEE. https://doi.org/10.1109/LACLO.2018.00060

Lockwood, J., \& Mooney, A. (2018). Computational Thinking in Secondary Education: Where does it fit? A systematic literary review. International Journal of Computer Science Education in Schools, 2(1), 41-60. https://doi.org/10.21585/ijcses.v2i1.26

Manrique-Losada, B., Gómez-Álvarez, M. C., \& González-Palacio, L. (2020). Estrategia de transformación para la formación en informática: hacia el desarrollo de competencias en educación básica y media para la Industria 4.0 en Medellín-Colombia. Revista Ibérica de Sistemas e Tecnologias de Informação, (39), 1-17. https://doi.org/10.170i3/risti.39.1-17

McHugh, M. L. (2012). Interrater reliability: the kappa statistic. Biochemia medica, 22(3), 276-282.

Integración del Pensamiento Computacional en la educación primaria y secundaria en Latinoamérica: una revisión sistemática de literatura. D. A. Quiroz-Vallejo, J.A. CarmonaMesa, A. Castrillón-Yepes y J.A. Villa-Ochoa. 
RED. Revista de Educación a Distancia. Núm. 68, Vol. 21. Artíc. 7, 30-Nov-2021 DOI: http://dx.doi.org/10.6018/red.485321

Papert, S. (1972). Teaching children to be mathematicians versus teaching about mathematics. International journal of mathematical education in science and technology, 3(3), 249-262. https://doi.org/10.1080/0020739700030306

Pérez, A. (2018). A framework for computational thinking dispositions in mathematics education. Journal for Research in Mathematics Education, 49(4), 424-461. https://doi.org/10.5951/jresematheduc.49.4.0424

Perković, L., Settle, A., Hwang, S., \& Jones, J. (2010, June). A framework for computational thinking across the curriculum. In Proceedings of the fifteenth annual conference on Innovation and technology in computer science education (pp. 123-127). https://doi.org/10.1145/1822090.1822126

Quiroz-Vallejo, D. A. (2020). Computational Thinking, Curriculum and Interdisciplinarity. An interview with Johannes Krugel. Unipluriversidad, 20(e2020110), 197-204. https://doi.org/10.17533/udea.unipluri.20.1.11

Rodrigues, R. S., Andrade, W. L., \& Campos, L. M. S. (2016, October). Can Computational Thinking help me? A quantitative study of its effects on education. In 2016 IEEE Frontiers in Education Conference (FIE) (pp. 1-8). IEEE. https://doi.org/10.1109/FIE.2016.7757409

Roig-Vila, R., \& Moreno-Isac, V. (2020). El pensamiento computacional en Educación. Análisis bibliométrico y temático. Revista de Educación a Distancia (RED), 20(63). https://doi.org/10.6018/red.402621

Rosales, V. A., Queiruga, C., Tzancoff, C. B., Kimura, I. M., \& Bartneche, M. B. (2017, September). Programming competitions in high school classrooms: RITA en RED. In 2017 XLIII Latin American Computer Conference (CLEI) (pp. 1-8). IEEE. https://doi.org/10.1109/CLEI.2017.8226399

Schneider, G., Bernardini, F., \& Boscarioli, C. (2020, October). Teaching CT through Internet of Things in High School: Possibilities and Reflections. In 2020 IEEE Frontiers in $\begin{array}{llll}\text { Education } \quad \text { Conference (FIE) } & \text { (pp. } & \text { 1-8). }\end{array}$ https://doi.org/10.1109/FIE44824.2020.9274184

Schneider, G., Bernardini, F., \& Boscarioli, C. (2020, October). Teaching CT through Internet of Things in High School: Possibilities and Reflections. In 2020 IEEE Frontiers in Education Conference (FIE) (pp. 1-8). IEEE. https://doi.org/10.21585/ijcses.v1i2.9

Shute, V. J., Sun, C., \& Asbell-Clarke, J. (2017). Demystifying computational thinking. Educational Research Review, 22, 142-158. https://doi.org/10.1016/j.edurev.2017.09.003

Sneider, C., Stephenson, C., Schafer, B., \& Flick, L. (2014). Computational thinking in high school science classrooms. The Science Teacher, 81(5), 53.

Integración del Pensamiento Computacional en la educación primaria y secundaria en Latinoamérica: una revisión sistemática de literatura. D. A. Quiroz-Vallejo, J.A. CarmonaMesa, A. Castrillón-Yepes y J.A. Villa-Ochoa. 
RED. Revista de Educación a Distancia. Núm. 68, Vol. 21. Artíc. 7, 30-Nov-2021 DOI: http://dx.doi.org/10.6018/red.485321

Souza, I. M. L., Andradre, W. L., \& Sampaio, L. M. R. (2019). Analyzing the Effect of Computational Thinking on Mathematics through Educational Robotics. Proceedings Frontiers in Education Conference, FIE, 2019-Octob. https://doi.org/10.1109/FIE43999.2019.9028384

Stephens M., Kadijevich D.M. (2019) Computational/Algorithmic Thinking. In: Lerman S. (eds) Encyclopedia of Mathematics Education. Springer, Cham. https://doi.org/10.1007/978-3-319-77487-9_100044-1

Tabesh, Y. (2017). Computational thinking: A 21st century skill. Olympiads in Informatics, 11(2), 65-70. https://doi.org/10.15388/ioi.2017.special.10

Tang, K. Y., Chou, T. L., \& Tsai, C. C. (2020). A Content Analysis of Computational Thinking Research: An International Publication Trends and Research Typology. Asia-Pacific Education Researcher, 29(1), 9-19. https://doi.org/10.1007/s40299-019-00442-8

Tang, X., Yin, Y., Lin, Q., Hadad, R., \& Zhai, X. (2020). Assessing computational thinking: A systematic review of empirical studies. Computers \& Education, 148, 103798. https://doi.org/10.1016/j.compedu.2019.103798

Villa-Ochoa, J. A., \& Castrillon-Yepes, A. (2020). Temas y tendencias de investigación en América Latina a la luz del pensamiento computacional en Educación Superior. In Políticas, Universidad e innovación: retos y perspectivas (pp. 235-248). https://doi.org/10.2307/j.ctv1dv0v33.14

von Wangenheim, C. (2019). SplashCode--A Board Game for Learning an Understanding of Algorithms in Middle School. Informatics in Education, 18(2), 259-280. https://doi.org/10.15388/infedu.2019.12

von Wangenheim, C. G., Alves, N. C., Rodrigues, P. E., \& Hauck, J. C. (2017). Teaching Computing in a Multidisciplinary Way in Social Studies Classes in School - A Case Study. International Journal of Computer Science Education in Schools, 1(2), 3-16. https://doi.org/10.21585/ijcses.v1i2.9

Weintrop, D., Beheshti, E., Horn, M., Orton, K., Jona, K., Trouille, L., \& Wilensky, U. (2016). Defining computational thinking for mathematics and science classrooms. Journal of Science Education and Technology, 25(1), 127-147. https://doi.org/10.1007/s10956-015$9581-5$

Wing, J. (2011). Computational thinking-What and why? The Link Magazine, June 23, 2015. Retrieved from http://www.cs.cmu.edu/link/research-notebook-computationalthinking-what-and-why

Wing, J. M. (2006). Computational thinking. Communications of the ACM, 49(3), 33-35. https://doi.org/10.1145/1118178.1118215

Integración del Pensamiento Computacional en la educación primaria y secundaria en Latinoamérica: una revisión sistemática de literatura. D. A. Quiroz-Vallejo, J.A. CarmonaMesa, A. Castrillón-Yepes y J.A. Villa-Ochoa. 
RED. Revista de Educación a Distancia. Núm. 68, Vol. 21. Artíc. 7, 30-Nov-2021 DOI: http://dx.doi.org/10.6018/red.485321

Wing, J. M. (2008). Computational thinking and thinking about computing. Philosophical Transactions of the Royal Society A: Mathematical, Physical and Engineering Sciences, 366(1881), 3717-3725. https://doi.org/10.1098/rsta.2008.0118

Wohlin, C., Runeson, P., Höst, M., Ohlsson, M. C., Regnell, B., \& Wesslén, A. (2012). Systematic literature reviews. In Experimentation in software engineering (pp. 45-54). Springer, Berlin, Heidelberg. https://doi.org/10.1007/978-3-642-29044-2_4

Xu, L. (2018). Identifying Strategies for Teaching Computational Thinking by Problem Solving and Self-Awareness. In T. Bastiaens, J. Van Braak, M. Brown, L. Cantoni, M. Castro, R. Christensen, G. Davidson-Shivers, K. DePryck, M. Ebner, M. Fominykh, C. Fulford, S. Hatzipanagos, G. Knezek, K. Kreijns, G. Marks, E. Sointu, E. Korsgaard Sorensen, J. Viteli, J. Voogt, P. Weber, E. Weippl \& O. Zawacki-Richter (Eds.), Proceedings of EdMedia: World Conference on Educational Media and Technology (pp. 2413-2418). Amsterdam, Netherlands: Association for the Advancement of Computing in Education (AACE). Recuperado de https://www.learntechlib.org/primary/p/185372/.

Zampieri M. T., \& Javaroni S. L. (2020). A Dialogue Between Computational Thinking And Interdisciplinarity Using Scratch Software. Uni-Pluriversidad, 20(1), 100-117. https://doi.org/10.17533/udea.unipluri.20.1.06

Zapata-Ros, M. (2019). Pensamiento computacional desenchufado. Education in the Knowledge Society (EKS), 20, 29. https://doi.org/10.14201/eks2019_20_a18

Zapata-Ros, M. (2018). Pensamiento computacional. Una tercera competencia clave. En M. Zapata-Ros [Ed] El pensamiento computacional como una nueva alfabetización en las culturas digitales. Murcia: Universidad de Murcia, 4-87.

Zhang, L. C., \& Nouri, J. (2019). A systematic review of learning computational thinking through Scratch in K-9. Computers and Education, 141(June), 103607. https://doi.org/10.1016/j.compedu.2019.103607

Integración del Pensamiento Computacional en la educación primaria y secundaria en Latinoamérica: una revisión sistemática de literatura. D. A. Quiroz-Vallejo, J.A. CarmonaMesa, A. Castrillón-Yepes y J.A. Villa-Ochoa. 